\title{
Feedback Gain in Multiple Antenna Systems
}

\author{
Srikrishna Bhashyam, Ashutosh Sabharwal and Behnaam Aazhang
}

This work was performed at the Department of Electrical and Computer Engineering, Rice University, Houston, Texas. Srikrishna Bhashyam (srikrishna@qualcomm.com) is currently with Qualcomm Inc.. Ashutosh Sabharwal (ashu@rice.edu) and Behnaam Aazhang (aaz@rice.edu) are with the Department of Electrical and Computer Engi-

neering (MS-366), Rice University, 6100, S. Main St., Houston, TX 77005. This work was supported in part by Nokia Corporation, Texas Advanced Technology Program, and National Science Foundation. 


\begin{abstract}
Multiple antenna transmission and reception have been shown to significantly increase the achievable data rates of wireless systems. However, most of the existing analysis assumes perfect or no channel information at the receiver and transmitter. The performance gap between these extreme channel assumptions is large and most practical systems lie in between. Therefore, it is important to analyze multiple antenna systems in the presence of partial channel information. In this paper, we upper bound the outage probability performance of multiple antenna systems with preamble-based channel estimation and quantized feedback. We design causal feedback and power control schemes to minimize this upper bound on outage probability. We consider the following practical issues in our analysis and design: (i) the channel information is imperfect both at the receiver and at the transmitter, and (ii) part of the total available resources for the system need to be used for estimation and feedback. Our results demonstrate that for block fading channels, sending a periodic preamble and causally receiving channel state information via a feedback channel can lead to substantial gains in the outage performance over any non-feedback scheme. Most of the gains achieved by perfect feedback can be achieved by very few bits of feedback. Furthermore, it is demonstrated that these outage probability gains can be translated into improvements in frame error rate performance of systems using space-time codes. Thus, implementing a power control, even at the cost of reduced spectral resources for the forward channel is beneficial for block fading channels.
\end{abstract}

\title{
I. INTRODUCTION
}

Multiple antenna transmission and reception has been shown to provide significant gains in achievable data rates over single antenna systems for fading channels [1-4]. Most of the information theoretic analysis assumes that either the receiver is completely aware of fading coefficients [1], or both the transmitter and receiver have perfect knowledge of the channel coefficients $[1,3,5]$, or neither the transmitter and receiver know the channel coefficients [2].

In [3], it has been shown that the outage performance with perfect channel information at the receiver and transmitter is significantly better compared to the case when only the receiver has perfect knowledge of the channel. However, practical systems seldom have perfect channel information. The channel has to be estimated at the receiver and fed back to the transmitter $^{1}$. Some important consequences of this are (i) the channel information is imperfect both at the receiver and at the transmitter, and (ii) part of the total available resources for the system need to be used for estimation and feedback. Since none of these effects have been considered in [3], the outage probabilities calculated are only loose lower bounds

\footnotetext{
${ }^{1}$ In slowly fading time-division duplex (TDD) systems, the channel measurement on the uplink can be used in the downlink.
} 
on outage performance of systems with more practical assumptions on channel information and feedback. Feedback of perfect channel information has also been shown to significantly improve capacity of fading channels in [5]. For single antenna systems, power control and adaptive signaling have been studied in [6] to improve the rate for a given average bit error probability using perfect channel information at the transmitter.

Some results pertaining to imperfect channel information at the receiver [7] and transmitter $[4,8,9]$ have also appeared. The effect of imperfect channel information at the receiver on capacity is studied in [7]. In [4] and [8], the effect of imperfect feedback on expected signal to noise ratio and capacity are studied and [9] studies the capacity of preamble-based systems with imperfect feedback. We study the outage performance of multiple antenna systems, instead of capacity, and consider the effect of imperfect channel information at the receiver as well as the transmitter and carefully account for the feedback resources in the total system design.

In this paper, we consider the outage performance of multiple antenna systems with preamble-based channel estimation and finite-rate quantized feedback under an average transmit power constraint. The resources used for the preamble and feedback are included as part of the total system resources ${ }^{2}$. We show that, in the presence of perfect channel information at the transmitter, the performance of power controlled space-time codes is much better than beamforming without power control for most cases of interest. Hence we limit our attention to power control without beamforming in this paper. First, we determine an upper bound on the performance of preamble-based systems with no feedback. Then, we determine the outage performance of a system with perfect channel information at the receiver and quantized feedback. Finally, we determine an upper bound on outage probability for preamble-based systems with quantized feedback. The upper bound on outage probability is then minimized over the choice of quantizer at the receiver and power control strategy at the transmitter. For 1-bit feedback, the jointly optimal quantizer and power control are evaluated analytically. For multiple bit feedback, the optimal power control strategy is analytically determined, and the quantizer is chosen by a numerical search. We show that significant gains can be achieved even with quantized feedback available from limited cooperation between the transmitter and receiver. In fact, the performance of a preamble-based system with 1-bit feedback is significantly better than a system with no feedback and perfect

\footnotetext{
${ }^{2}$ Both transmitter and receiver are equipped with their own power source, hence the transmitter power constraint is not affected by the implementation of the feedback.
} 
channel information at the receiver.

Once we have evaluated the outage probability performance, we demonstrate how similar gains can be achieved in terms of the frame error rate of a multiple antenna system that uses space-time codes. The quantizer and power control strategies are designed for two illustrative space-time codes from [10] and [11]. We limit our attention to the practically important case of multiple transmit and single receive antenna. Due to size and battery power limitation, most of the current hand-held devices can accommodate only one antenna. Therefore, the proposed system can be applied to communication from base-station to mobile. Results pertaining to multiple receive antennas will be presented elsewhere. The effect of delay in the feedback path is also not considered in this paper. Delayed feedback can still be useful [12] if the channel is slowly time-varying, and its effect can be studied by understanding the correlation properties of the channel over the duration of the delay.

The rest of the paper is organized as follows. In Section II, we briefly describe the system and channel model, and in Section III, we review existing outage results with perfect channel information to motivate the rest of the paper. In Section IV, we evaluate the outage for preamble-based systems with quantized feedback and consider the effect of feedback errors in Section V. In Section VI, we demonstrate the use of feedback and power control with space-time codes. The numerical and simulation results are presented in Section VII and the conclusions are in Section VIII.

\section{System MOdeL}

We consider the independent and identically distributed (i.i.d.) block fading channel model of $[1,2]$. For a multiple antenna system with $M$ transmit antennas and one receive antenna, this model leads to the following complex baseband representation of the received signal.

$$
y=\mathbf{h} \mathbf{x}+w,
$$

where $\mathbf{x}$ is the $M \times 1$ vector of transmitted symbols, $\mathbf{h}$ is the $1 \times M$ channel vector, $w$ is the complex circularly symmetric additive white Gaussian noise and $y$ is the received signal. As per the model, the channel vector is assumed to be constant for each transmission block of $T$ symbols and changes independently from one block to another. The elements of the channel vector $\mathbf{h}$ and the noise $w$ are circularly symmetric complex Gaussian distributed with zero mean and variance 1 . The elements of $\mathbf{h}$ are also uncorrelated. The average power constraint on the transmissions can be expressed as $\mathbb{E}\left[\mathbf{x}^{H} \mathbf{x}\right] \leq P$. 


\section{Outage Probability with Perfect Channel Information}

In this section, we will review some of the results on outage probability with perfect channel state information $[1,13,14]$. We will concentrate on the results for systems with only one receive antenna. These results act as lower bounds on the performance of practical systems with imperfect channel information evaluated in this paper. They also motivate the design of the power control and feedback schemes used in the rest of the paper. For simplicity, we will use the acronym CSIR to denote channel state information at the receiver, and CSIT to denote channel state information at the transmitter.

\section{A. Perfect CSIR}

We first consider the case where the channel is known exactly at the receiver and not known at the transmitter. In this case, for a given channel realization $\mathbf{h}$ that is randomly chosen and with an average power constraint $P$, we can restrict ourselves to a zero-mean complex Gaussian input to maximize the mutual information between the input $\mathbf{x}$ and output $y$ [1]. Given a choice of input such that $\mathbb{E}\left[\mathbf{x x}^{H}\right]=\mathbf{Q}$, the mutual information (in nats $/ \mathrm{s} / \mathrm{Hz}$ ) is given by

$$
I(\mathbf{x} ; y \mid \mathbf{h})=\log \left(1+\mathbf{h} \mathbf{Q} \mathbf{h}^{H}\right)
$$

The minimum outage probability is then obtained by optimizing over $\mathbf{Q}$, and is given by

$$
\Pi_{\text {out }}(R, P)=\inf _{\mathbf{Q}: \mathbf{Q} \geq 0, \operatorname{tr}(\mathbf{Q}) \leq P} \operatorname{Prob}\left(\log \left(1+\mathbf{h}_{t} \mathbf{Q h}_{t}^{H}\right)<R\right),
$$

where $\mathbf{h}_{t}$ corresponds to the first $t$ elements of $\mathbf{h}$. For the single transmit and receive antenna case, it is easy to see that $\mathbf{Q}$ should be equal to $P$. For the multiple transmit antenna case with one receive antenna, the conjecture in [1] is that the optimal $\mathbf{Q}$ is diagonal with $t$ $(1 \leq t \leq M)$ of the diagonal elements equal to $P / t$ and the other elements equal to zero. With this conjecture, the outage probability is given by

$$
\Pi_{\text {out }}(R, P)=\min _{1 \leq t \leq M} \operatorname{Prob}\left(\log \left(1+\frac{P}{t} \mathbf{h}_{t} \mathbf{h}_{t}^{H}\right)<R\right) .
$$

Since $\mathbf{h} \sim \mathcal{C N}(\mathbf{0}, \mathbf{I})$, it implies that the instantaneous channel signal to noise ratio, $\gamma=\mathbf{h}_{t} \mathbf{h}_{t}^{H}$, is chi-squared distributed with $2 t$ degrees of freedom, and its probability density function is given by

$$
p_{\gamma}(\gamma)=\frac{1}{\Gamma(t)} \gamma^{t-1} e^{-\gamma}
$$

where $\Gamma($.$) denotes the Gamma function. Thus, the outage probability can be obtained as$ $\min _{1 \leq t \leq M} \operatorname{Prob}\left(\gamma \leq t\left(e^{R}-1\right) / P\right)$. 


\section{B. Perfect CSIR and CSIT}

With perfect CSIT, the transmitter can use the knowledge of the channel realization in choosing Q. It was shown in [14], that the optimal transmission scheme for a general multiple transmit and receive antenna case is an optimal power control scheme concatenated with an optimal beamformer. The optimal beamforming technique directs the transmission along the direction given by $\mathbf{h}^{H} / \sqrt{\mathbf{h} \mathbf{h}^{H}}$. The optimal power control scheme determines the power along this direction, $P(\mathbf{h}, R)$, based on the knowledge of the channel $\mathbf{h}$ and the transmission rate $R$. The average power constraint is imposed by ensuring that $\mathbb{E}[P(\mathbf{h}, R)] \leq P$.

The received signal with optimal beamforming and power control is given by

$$
y=\sqrt{P(\mathbf{h}, R)} \sqrt{\mathbf{h h}^{H}} x+w
$$

where $y$ is the signal at the receiver antenna, $w$ is the additive complex Gaussian noise, $x$ is the transmitted signal along the eigenvector direction (scalar in this case), $M$ is the number of transmit antennas and $P(\mathbf{h}, R)$ is the power of the transmission. The mutual information is given by

$$
I(x ; y \mid \mathbf{h})=\log \left(1+P(\mathbf{h}, R) \mathbf{h h}^{H}\right) .
$$

To avoid outage at a particular rate $R$, the power should be chosen as

$$
P(\mathbf{h}, R)=\frac{\left(e^{R}-1\right)}{\gamma},
$$

where $\gamma=\mathbf{h h}^{H}$. Since the power control should satisfy the average power constraint $\mathbb{E}[P(\mathbf{h}, R)] \leq P$, the smallest threshold $\gamma_{0}$ for $\gamma$ is chosen such that the power constraint given by

$$
\int_{\gamma_{0}}^{\infty}\left(\frac{e^{R}-1}{\gamma}\right) p_{\gamma}(\gamma) d \gamma \leq P
$$

is satisfied. Thus, for a given rate, we find the threshold above which outage can be avoided while satisfying the power constraint. The minimum outage probability is, therefore, calculated as $\mathcal{P}\left(\gamma<\gamma_{0}\right)$.

The gains from beamforming and power control can be individually identified. The maximum mutual information with power control alone is given by

$$
I(x ; y \mid \mathbf{h})=\log \left(1+\frac{P(\mathbf{h}, R)}{M} \mathbf{h h}^{H}\right),
$$


where the power control is chosen as

$$
P(\mathbf{h}, R)=\frac{M\left(e^{R}-1\right)}{\gamma}
$$

with the average power constraint imposed as above. The minimum outage probability, is calculated as $\mathcal{P}\left(\gamma<\gamma_{0}\right)$, with

$$
\int_{\gamma_{0}}^{\infty}\left(\frac{e^{R}-1}{\gamma}\right) p_{\gamma}(\gamma) d \gamma \leq \frac{P}{M}
$$

The maximum mutual information with beamforming alone is given by

$$
I(x ; y \mid \mathbf{h})=\log \left(1+P \mathbf{h h}^{H}\right)
$$

The outage performance of all of the above three scenarios: power control and beamforming, power control only, and beamforming only are compared with each other and with the perfect CSIR case in Figures 2 and 3. From the above expressions and the result in Figures 2 and 3, we see that significant gains in outage performance can be achieved with feedback. Specifically, we can make the following observations:

1. For multiple antenna systems, even zero outage can be achieved [3] with perfect CSIR and CSIT (perfect feedback) for nonzero transmission rates. With power control and beamforming, zero outage is achieved for rates less than $\log (1+P(M-1))$. With power control only, zero outage can be achieved for rates less than $\log (1+P(M-1) / M)$. However, zero outage can never be achieved without feedback or with beamforming alone irrespective of the number of transmit antennas. Even when the outage is non-zero, the outage performance of all systems with feedback are significantly lower than the system with no feedback (for example, see outage in Figure 3 for $R=5 \mathrm{bits} / \mathrm{s} / \mathrm{Hz}$ ); see Section VII for more details.

2. Power control provides significant gains in performance compared to the no feedback case. The decay in outage probability with increasing $P$ is much faster with power control. This is very significant as long as the number of antennas is not too large (for example, in Figure 2 where $M=4$, to achieve an outage of $10^{-4}$, we need $17-18 \mathrm{~dB}$ of power with just perfect CSIR but we need only $6 \mathrm{~dB}$ with power control.).

3. Beamforming provides a constant gain of $\log (M) \mathrm{dB}$, where $M$ is the number of transmit antennas, and does not improve the decay rate of outage probability with $P$. This gain becomes more significant than the gain from only power control for large number of antennas, but it requires substantially more feedback information ( $M$ parameters instead of 1 ). 
Thus, considering the significant potential performance improvement provided by feedback, it now becomes important to understand and evaluate the gains achievable when perfect channel feedback is not possible and limited resources are available for feedback. In the next section, we evaluate the outage performance in the presence of partial feedback, and illustrate significant achievable gain. Since we concentrate on systems with limited feedback resources (mainly 1 or 2 bits of feedback), we restrict our attention to power control only. Thus, for the rest of paper, we will use the outage probability with power control only as the lower bound for comparison. Beamforming can be taken advantage of when more feedback is available. For reasonably small number of antennas and limited feedback, the potential gain from power control is significant. The use of beamforming in the presence of complete and partial feedback are studied in $[4,8,15-17]$.

\section{Outage Probability with Imperfect Channel Information}

Practical wireless communication systems seldom have perfect channel information at the transmitter or receiver. Channel information is usually estimated and hence has an error associated with it. Moreover, part of the available resources may have to be spent to obtain this channel information. In this section, we will analyze the effect of imperfect channel information on the outage performance of multiple antenna systems. We will analyze the following three cases: preamble-based CSIR with no feedback, perfect CSIR with quantized feedback and preamble-based CSIR with quantized feedback. The first case studies the effect of channel estimation error at the receiver in the absence of feedback. The second case studies the effect of partial (quantized) channel information at the transmitter assuming perfect CSIR. Finally, the analysis methods of the first two simpler problems are combined to obtain an outage probability bound for a system with imperfect CSIR and quantized feedback.

\section{A. Preamble-based CSIR with No Feedback}

In this section, we will derive an upper bound for the outage probability of multiple antenna systems using preamble-based channel estimation schemes. For an $M$ transmit antenna system with one receive antenna, there are $M$ unknown channel coefficients to be estimated. Thus, at least $M$ independent measurements are needed to obtain finite variance estimates of the $M$ unknown channel coefficients. A simple preamble-based method of obtaining channel estimates is to transmit the $i^{\text {th }}$ preamble symbol over the $i^{\text {th }}$ antenna during the $i^{\text {th }}$ symbol 
duration $(1 \leq i \leq M)$. Thus, after $M$ transmissions, the receiver has an estimate of all the channel coefficients with finite variance. In order to reduce estimation error variance, we can either increase the preamble duration or use more power during the preamble transmission than during data transmission. Since the mutual information decreases logarithmically in power and linearly in time, we choose to transmit the least number of symbols during the preamble and adjust the power to improve estimation.

Let the power of each transmitted preamble symbol be $P_{t} / M$. We choose the channel estimate to be the minimum mean squared error (MMSE) channel estimate. It is given by [18]

$$
\hat{\mathbf{h}}=\frac{\sqrt{P_{t} / M}}{P_{t} / M+1}\left(\sqrt{\frac{P_{t}}{M}} \mathbf{h}+\mathbf{n}\right),
$$

where $\mathbf{n}$ is the $M \times 1$ complex Gaussian vector of the additive noise observed in the channel during the $M$ preamble symbol periods. The MMSE estimate has the following two properties:

$$
E[\mathbf{h} \mid \hat{\mathbf{h}}]=\hat{\mathbf{h}},
$$

and

$$
E\left[\|\mathbf{h}-\hat{\mathbf{h}}\|^{2} \mid \hat{\mathbf{h}}\right]=\frac{1}{\frac{P_{t}}{M}+1}=\gamma_{p}^{2} .
$$

The property in (15) is important and will be used later in order to derive the upper bound on outage probability.

From the system model, we have

$$
y=\mathbf{h} \mathbf{x}+w=\hat{\mathbf{h}} \mathbf{x}+(\mathbf{h}-\hat{\mathbf{h}}) \mathbf{x}+w=\hat{\mathbf{h}} \mathbf{x}+\hat{w} .
$$

We will derive a lower bound for $I(\mathbf{x} ; y \mid \hat{\mathbf{h}})$ and use that to get the upper bound on outage probability. This bound is derived in a manner similar to that in [19] where single antenna fading channels with imperfect channel information are studied. The analysis in [7] is also related to our analysis. First, we restrict ourselves to the use of Gaussian codebooks, i.e., we choose $\mathbf{x}$ to be zero-mean complex Gaussian with independent and identically distributed components of variance $P_{d} / M$. Any upper bound on the achievable outage probability in this case with also be an upper bound on the minimum outage probability achievable with arbitrary codebooks.

Then, we can show that

$$
E\left[\hat{w} \mathbf{x}^{H} \mid \hat{\mathbf{h}}\right]=0
$$


in the following manner using (15) and the fact that $\mathbf{x}$ is independent of $(\mathbf{h}, \hat{\mathbf{h}})$.

$$
\begin{aligned}
E\left[\hat{w} \mathbf{x}^{H} \mid \hat{\mathbf{h}}\right] & =E\left[((\mathbf{h}-\hat{\mathbf{h}}) \mathbf{x}+w) \mathbf{x}^{H} \mid \hat{\mathbf{h}}\right] \\
& =E\left[(\mathbf{h}-\hat{\mathbf{h}}) \mathbf{x} \mathbf{x}^{H} \mid \hat{\mathbf{h}}\right] \\
& =E[(\mathbf{h}-\hat{\mathbf{h}}) \mid \hat{\mathbf{h}}] \cdot E\left[\mathbf{x} \mathbf{x}^{H} \mid \hat{\mathbf{h}}\right] \\
& =0 \cdot E\left[\mathbf{x} \mathbf{x}^{H} \mid \hat{\mathbf{h}}\right] \\
& =0
\end{aligned}
$$

We can also lower bound the mutual information, $I(\mathbf{x} ; y \mid \hat{\mathbf{h}})$, as follows.

$$
\begin{aligned}
I(\mathbf{x} ; y \mid \hat{\mathbf{h}}) & =H(\mathbf{x} \mid \hat{\mathbf{h}})-H(\mathbf{x} \mid y, \hat{\mathbf{h}}) \\
& =H(\mathbf{x} \mid \hat{\mathbf{h}})-H(\mathbf{x}-\mathbf{g} y \mid y, \hat{\mathbf{h}}) \\
& \geq H(\mathbf{x} \mid \hat{\mathbf{h}})-H(\mathbf{x}-\mathbf{g} y \mid \hat{\mathbf{h}}) .
\end{aligned}
$$

In the second step, the addition of any term dependent only on $y$ and $\hat{\mathbf{h}}$ will not alter the entropy. In the above case we use $\mathbf{g} y$, where $\mathbf{g}$ is a vector that we will choose later. The inequality in the third step follows from the fact that

$$
H(\mathbf{x}-\mathbf{g} y \mid y, \hat{\mathbf{h}}) \leq H(\mathbf{x}-\mathbf{g} y \mid \hat{\mathbf{h}})
$$

For any zero-mean random vector $\mathbf{x}$ with $E\left[\mathbf{x x}^{H}\right]=Q$, we have [1]

$$
H(\mathbf{x}) \leq \log (\operatorname{det}(\pi e Q))
$$

with equality when $\mathbf{x}$ is proper complex Gaussian. Therefore, we get

$$
I(\mathbf{x} ; y \mid \hat{\mathbf{h}}) \geq \log (\operatorname{det}(\pi e Q))-\log \left(\operatorname{det}\left(\pi e E\left[(\mathbf{x}-\mathbf{g} y)(\mathbf{x}-\mathbf{g} y)^{H} \mid \hat{\mathbf{h}}\right]\right)\right)
$$

We scale the lower bound by $(T-M) / T$ in order to account for the preamble symbols used to obtain the channel estimate.

$$
I(\mathbf{x} ; y \mid \hat{\mathbf{h}}) \geq \frac{T-M}{M}\left[\log (\operatorname{det}(\pi e Q))-\log \left(\operatorname{det}\left(\pi e E\left[(\mathbf{x}-\mathbf{g} y)(\mathbf{x}-\mathbf{g} y)^{H} \mid \hat{\mathbf{h}}\right]\right)\right)\right]
$$

Our final step in deriving the bound is to choose the appropriate $\mathbf{g}$ to use in (21). We choose $\mathbf{g}$ to be the MMSE estimator of $\mathbf{x}$ from $y$ given $\hat{\mathbf{h}}$.

$$
\mathbf{g}=\frac{\frac{P_{d}}{M} \hat{\mathbf{h}}}{\frac{P_{d}}{M} \hat{\mathbf{h}}^{H}+E\left[|\hat{w}|^{2} \mid \hat{\mathbf{h}}\right]} .
$$


For this choice of $\mathbf{g}$, we get

$$
E\left[(\mathbf{x}-\mathbf{g} y)(\mathbf{x}-\mathbf{g} y)^{H} \mid \hat{\mathbf{h}}\right]=Q\left(I-\frac{\frac{P_{d}}{M} \hat{\mathbf{h}}^{H} \hat{\mathbf{h}}}{\frac{P_{d}}{M} \hat{\mathbf{h}}^{H}+E\left[|\hat{w}|^{2} \mid \hat{\mathbf{h}}\right]}\right) .
$$

Substituting this back in (21), we have the lower bound

$$
I(\mathbf{x} ; y \mid \hat{\mathbf{h}}) \geq \frac{T-M}{T} \log \left(1+\frac{P_{d} \hat{\mathbf{h}}^{H}}{M\left(1+\gamma_{p}^{2} P_{d}\right)}\right) .
$$

The details of substitution of $\mathbf{g}$ in (21) to get the lower bound are in Appendix A.

The lower bound on the mutual information translates into an upper bound for the outage probability as follows:

$$
\Pi_{\text {out }}(R, P) \leq \operatorname{Prob}\left(\frac{T-M}{T} \log \left(1+\frac{P_{d}}{M\left(1+\gamma_{p}^{2} P_{d}\right)} \hat{\mathbf{h}} \hat{\mathbf{h}}^{H}\right)<R\right) .
$$

The upper bound can be minimized over the data power $P_{d}$ to obtain a tighter upper bound. Using the relation, $P_{t}+(T-M) P_{d}=P$, the value of $P_{d}$ that minimizes the bound can be shown to satisfy the quadratic equation $a P_{d}^{2}+b P_{d}+c=0$, where $a=(T-M)(T-2 M)$, $b=-2(T-M)(T P+M)$ and $c=(T P+M) T P$. From the two solutions to this quadratic equation, we choose the solution that yields positive solutions to the preamble and data powers, i.e., $P_{t}, P_{d} \geq 0$.

This upper bound on outage probability is calculated in Section VII and the outage probability with perfect CSIR acts as the lower bound. The upper bound is calculated for the following cases: (i) equal training and data power, and (ii) optimized training and data power. The results show that the proper choice of training and data power significantly im-

proves the outage performance. It can also be seen that for a fixed $M$ (number of transmit antennas), the upper bound approaches the perfect CSIR performance as the block length $T$ increases [9].

\section{B. Perfect CSIR and Quantized Feedback}

In this section, we evaluate the outage probability of a multiple transmit antenna system with perfect CSIR and quantized feedback. As mentioned in Section III-B, we concentrate on power control. Therefore, the feedback is obtained by quantizing $\gamma=\mathbf{h h}^{H}$. Specifically, the use of optimal power control without beamforming and a choice of $\mathbf{Q}=\mathbf{I}$, gives

$$
y=\sqrt{\frac{P(\mathbf{h}, R)}{M}} \mathbf{h} \mathbf{x}+w
$$


and

$$
I(\mathbf{x} ; y \mid \mathbf{h})=\log \left(1+\frac{P(\mathbf{h}, R)}{M} \mathbf{h h}^{H}\right) .
$$

The optimal power control in Section III-B assumes the exact knowledge of $\gamma$. In this section, we will use quantized $\gamma$ and use a simple form of power control $P(\mathbf{h}, R)=\operatorname{Pf}(\gamma)$, where $f(\gamma)$ is a function that captures the effect of quantization and power control. Figure 1 describes the function $f(\gamma)$ and introduces the various quantizer and power control parameters. We denote the $2^{q}-1$ thresholds of the quantizer as $\gamma_{0}, \gamma_{1}, \ldots, \gamma_{2^{q}-2}$ with $\gamma_{0} \leq \gamma_{1} \leq \ldots \leq \gamma_{2^{q}-2}$. The $B=2^{q}$ quantizer bins are $\left[0, \gamma_{0}\right),\left[\gamma_{0}, \gamma_{1}\right), \ldots,\left[\gamma_{B-2}, \infty\right)$. The power control scheme is the piecewise constant function that determines the transmitted power corresponding to each of these bins. The transmit power corresponding the $B$ bins are denoted by $\alpha_{1} P, \alpha_{2} P$, $\ldots, \alpha_{B} P$, where $P$ is the average power constraint. In order to satisfy the power constraint, $f(\gamma)$ should be chosen such that $\mathbb{E}[f(\gamma)]=1$. Substituting for $P(\mathbf{h}, R)$ in (25), the outage probability is written as

$$
\Pi_{\text {out }}(R, P)=\operatorname{Prob}\left(\log \left(1+\frac{P f(\gamma)}{M} \mathbf{h h}^{H}\right)<R\right) .
$$

The parameters of $f(\gamma)$, i.e., the thresholds $\left\{\gamma_{i}\right\}_{i=0}^{2^{q}-2}$ for the quantizer and the associated power levels $\left\{\alpha_{i}\right\}_{i=1}^{2^{q}}$, are chosen to minimize outage while satisfying the average power constraint. For the 1-bit feedback case $(q=1)$, the optimal quantizer and power control can be obtained analytically. For a general $q>1$, it seems impossible to obtain the jointly optimal choice of all the parameters analytically. However, we can find the optimal power control for a fixed quantizer, i.e., fixed $\left\{\gamma_{i}\right\}_{i=0}^{2^{q}-2}$. The best quantizer is chosen by searching over a search set of quantizers. The optimal power control scheme for a $q$-bit quantizer will be described in Section IV-B.1 and the jointly optimal power control and quantizer for 1-bit feedback will be presented in Section IV-B.2.

\section{B.1 Power Control for Quantized Feedback}

In this section, we determine the optimal power control scheme given $q$-bit quantized feedback of $\gamma$, i.e., we find the optimal $\left\{\alpha_{i}\right\}_{i=1}^{2^{q}}$ given $\left\{\gamma_{i}\right\}_{i=0}^{2^{q}-2}$.

First, we note that the optimal power control scheme has the following properties:

1. $\alpha_{i}>0$ only if zero outage is achievable in all bins $\left[\gamma_{i-1}, \gamma_{i}\right),\left[\gamma_{i}, \gamma_{i+1}\right), \ldots,\left[\gamma_{B-2}, \infty\right)$. This property states that since it requires lower power to avoid outage for larger $\gamma$, it is always better to allocate power to the bins corresponding to larger SNR, $\gamma$, before using any power for the bins corresponding to lower SNR, $\gamma$. 
2. For $i>1$, the optimal $\alpha_{i}$ is

$$
\alpha_{i}=\min \left(\frac{\left(e^{R}-1\right) M}{P \gamma_{i-2}}, \frac{P_{r e m}}{P}\right),
$$

where $P_{\text {rem }}$ is the power remaining under the power constraint after $\alpha_{j}$ has been determined for all $j>i$. For $i=1, \alpha_{1}=P_{r e m} / P$. This property states that we need to allocate the minimum power needed to avoid outage in each bin. When there is not enough power to avoid outage completely, we should use all of the remaining power.

Then, using the above properties, the optimal power control scheme is obtained using the following procedure.

Step 1. Set $P_{r e m}=P$.

Step 2. Set $j=B$.

Step 3. Find $\alpha_{j}$ using

$$
\alpha_{j}=\min \left(\frac{\left(e^{R}-1\right) M}{P \gamma_{j-2}}, \frac{P_{r e m}}{P}\right) .
$$

Step 4. Set $P_{\text {rem }}=P_{\text {rem }}-\alpha_{j} P$.

Step 5. While $j>1$, set $j=j-1$ and repeat steps 3 and 4 .

Finally, we note an interesting fact that for the above quantizer, the outage can never be zero with finite number of bits used for feedback. This is because the power allocated to each quantizer bin has to be finite to satisfy the power constraint since the probability of each quantizer bin is nonzero. As a result, there is always a nonzero positive number $\gamma_{\min }$ such that for $\gamma \leq \gamma_{\min }$ there is always an outage and the outage probability, which is $\operatorname{Prob}\left(\gamma \leq \gamma_{\min }\right)$, is nonzero. We conjecture that this result is true for an arbitrary quantizer.

\section{B.2 Optimal Quantizer and Power Control for 1-bit Feedback}

For 1-bit feedback, the function $f(\gamma)$ can be written as

$$
f(\gamma)=\left\{\begin{array}{ll}
\alpha_{1} & \gamma \leq \gamma_{0} \\
\alpha_{2} & \gamma>\gamma_{0}
\end{array} .\right.
$$

Using (26), the outage probability can be written as

$$
\Pi_{\text {out }}=\operatorname{Prob}\left(\gamma f(\gamma) \leq \frac{\left(e^{R}-1\right) M}{P}\right) .
$$


We can rewrite the above outage probability in terms of the cumulative distribution function of $\gamma$ by considering the two possible regions, $\alpha_{1}>\alpha_{2}$ and $\alpha_{1} \leq \alpha_{2}$, separately. When $\alpha_{1}>\alpha_{2}$,

$$
\Pi_{\text {out }}= \begin{cases}\operatorname{Prob}\left[\gamma \leq \frac{z}{\alpha_{1}}\right] & z \leq \alpha_{2} \gamma_{0} \\ \operatorname{Prob}\left[\gamma \leq \frac{z}{\alpha_{1}}\right]+\operatorname{Prob}\left[\gamma_{0} \leq \gamma \leq \frac{z}{\alpha_{2}}\right] & \alpha_{2} \gamma_{0}<z \leq \alpha_{1} \gamma_{0} \\ \operatorname{Prob}\left[\gamma \leq \frac{z}{\alpha_{2}}\right] & z>\alpha_{1} \gamma_{0}\end{cases}
$$

where $z=\left(e^{R}-1\right) M / P$. When $\alpha_{1} \leq \alpha_{2}$,

$$
\Pi_{\text {out }}= \begin{cases}\operatorname{Prob}\left[\gamma \leq \frac{z}{\alpha_{1}}\right] & z \leq \alpha_{1} \gamma_{0} \\ \operatorname{Prob}\left[\gamma \leq \gamma_{0}\right] & \alpha_{1} \gamma_{0}<z \leq \alpha_{2} \gamma_{0} \\ \operatorname{Prob}\left[\gamma \leq \frac{z}{\alpha_{2}}\right] & z>\alpha_{2} \gamma_{0}\end{cases}
$$

The power constraint is

$$
\alpha_{1} \operatorname{Prob}\left[\gamma \leq \gamma_{0}\right]+\alpha_{2}\left(1-\operatorname{Prob}\left[\gamma \leq \gamma_{0}\right]\right) \leq 1
$$

The following two observations simplify the derivation of minimum outage probability.

1. For $\alpha_{1}>\alpha_{2}$, the optimal $\alpha_{1}, \alpha_{2}$ and $\gamma_{0}$ will satisfy $z \leq \alpha_{2} \gamma_{0}$, i.e., among the three regions of $z$ in (30) the optimal solution results in $z \leq \alpha_{2} \gamma_{0}$. This is because, if $z$ were in any other region, property (i) of the optimal power control in Section IV-B.1 would be violated.

2. For the region $\alpha_{1} \leq \alpha_{2}$, it is sufficient to consider the case where $\alpha_{1}=0$. This follows from the simple observation that it requires higher power to avoid outage for lower $\gamma$. Therefore, there is no reason to use any nonzero $\alpha_{1}$ which is lesser than $\alpha_{2}$ since it would not decrease the outage. This also follows from property (i) of the optimal power control scheme in Section IV-B.1.

Therefore, we obtain the following two solutions for minimum outage probability in the two regions and choose the minimum of the two solutions as the overall minimum outage probability.

1. For the case where $\alpha_{1}>\alpha_{2}$, obtain the minimum of $P\left[\gamma \leq z / \alpha_{1}\right]$ under the constraints $z \leq \alpha_{2} \gamma_{0}$ and $\alpha_{1} \operatorname{Prob}\left[\gamma \leq \gamma_{0}\right]+\alpha_{2}\left(1-\operatorname{Prob}\left[\gamma \leq \gamma_{0}\right]\right) \leq 1$ 
2. For the case where $\alpha_{1}=0$, choose $\alpha_{2}$ and $\gamma_{0}$ such that $z=\alpha_{2} \gamma_{0}$ and $\alpha_{2}\left(1-\mathcal{P}\left[\gamma \leq \gamma_{0}\right]\right)=1$. The details of the solution are given in Appendix B and the numerical calculations are provided in Section VII.

\section{Preamble-based CSIR and Quantized Feedback}

In this section, we will combine the analysis techniques from Section IV-A and Section IV-B, and obtain an upper bound on the minimum outage probability of preamble-based multiple antenna systems with quantized feedback. Thus, we include the effects of imperfect channel estimation both at the transmitter and at the receiver. First, we upper bound the outage probability for a preamble-based system with a fixed quantizer and power control scheme. Then, we minimize this upper bound over the possible quantizers and power control schemes. As in Section IV-B, the minimization can be done analytically for the 1-bit feedback case. For multiple bit feedback, the optimal power control scheme will be found only for a fixed quantizer and the quantizer will be chosen from a search set of quantizers.

Let the power of each transmitted preamble symbol be $P_{t} / M$. Then, the average power available for data transmission is $P_{d}=\left(P T-P_{t}\right) /(T-M)$. The actual power of each data frame can be written as $P_{d} f(\gamma)$, where $f(\gamma)$ is as defined in Section (IV-B). Using the upper bound from equation (23), we can write

$$
\Pi_{\text {out }}(R, P) \leq \operatorname{Prob}\left(\frac{T-M-q}{T} \log \left(1+\frac{P_{d} f(\gamma)}{M\left(1+\gamma_{p}^{2} P_{d} f(\gamma)\right)} \gamma\right)<R\right),
$$

where $\gamma=\hat{\mathbf{h}} \hat{\mathbf{h}}^{H}$ and the scaling $(T-M-q) / T$ accounts for both the preamble and feedback resources. This upper bound has to be minimized over the possible choices of $f(\gamma)$, which includes the quantizer and power control parameters, and $P_{t}$.

\section{C.1 Power Control for Quantized Feedback}

The optimal power control scheme given a quantizer is found similar to the approach in Section IV-B.1. The only modifications are in steps 1 and 3. This is because: (i) some power $P_{t}$ is used for training, and (ii) the mutual information for a preamble-based system in (23) is different from (26). The modified algorithm is given as:

Step 1. Set $P_{\text {rem }}=\left(P T-P_{t}\right) /(T-M-q)$.

Step 2. Set $j=B$. 
Step 3. Find $\alpha_{j}$ using

$$
\alpha_{i}=\min \left(\frac{M}{\left(\frac{\gamma}{e^{R^{\prime}}-1}-\gamma_{p}^{2}\right) P_{d}}, \frac{P_{r e m}}{P}\right)
$$

Step 4. Set $P_{\text {rem }}=P_{\text {rem }}-\alpha_{j} P$.

Step 5. While $j>1$, set $j=j-1$ and repeat steps 3 and 4 .

Similar to the perfect CSIR case with 1-bit feedback, the outage can never be zero in this case.

\section{C.2 Optimal Quantizer and Power Control with 1-bit Feedback}

For 1-bit feedback and a fixed training power, the minimization can be solved very similar to the way it was solved for perfect CSIR. In this section, we describe the key adjustments needed, with the details given in Appendix C.

The outage probability in this case is upper bounded as

$$
\Pi_{\text {out }} \leq \operatorname{Prob}\left(\frac{f(\gamma)}{\frac{M}{P_{d}}+\gamma_{p}^{2} f(\gamma)} \gamma \leq\left(e^{R^{\prime}}-1\right)\right)=\operatorname{Prob}\left(f_{1}(\gamma) \gamma \leq\left(e^{R^{\prime}}-1\right)\right)
$$

where

$$
R^{\prime}=\frac{R T}{T-M-1}
$$

and

$$
f_{1}(\gamma)=\frac{f(\gamma)}{\frac{M}{P_{d}}+\gamma_{p}^{2} f(\gamma)}=\left\{\begin{array}{cc}
\beta_{1} & \gamma \leq \gamma_{0} \\
\beta_{2} & \gamma>\gamma_{0}
\end{array}\right.
$$

with

$$
\beta_{i}=\frac{\alpha_{i}}{\frac{M}{P_{d}}+\gamma_{p}^{2} \alpha_{i}}
$$

Using $\beta_{1}$ and $\beta_{2}$ instead of $\alpha_{1}$ and $\alpha_{2}$, we can rewrite the outage probability in terms of the cumulative distribution of $\gamma$ for the two regions, $\beta_{1}>\beta_{2}$ and $\beta_{1} \leq \beta_{2}$. It is also clear that $\beta_{1}>\beta_{2}$ implies that $\alpha_{1}>\alpha_{2}$ and vice versa. Therefore, we can still use the same regions, $\alpha_{1}>\alpha_{2}$ and $\alpha_{1} \leq \alpha_{2}$, as in Section IV-B.2. When $\alpha_{1}>\alpha_{2}$,

$$
\Pi_{\text {out }} \leq \begin{cases}\operatorname{Prob}\left[\gamma \leq \frac{z}{\beta_{1}}\right] & z \leq \beta_{2} \gamma_{0} \\ \operatorname{Prob}\left[\gamma \leq \frac{z}{\beta_{1}}\right]+\operatorname{Prob}\left[\gamma_{0} \leq \gamma \leq \frac{z}{\beta_{2}}\right] & \beta_{2} \gamma_{0}<z \leq \beta_{1} \gamma_{0} \\ \operatorname{Prob}\left[\gamma \leq \frac{z}{\beta_{2}}\right] & z>\beta_{1} \gamma_{0}\end{cases}
$$


where $z=\left(e^{R^{\prime}}-1\right)$. When $\alpha_{1} \leq \alpha_{2}$,

$$
\Pi_{\text {out }} \leq \begin{cases}\operatorname{Prob}\left[\gamma \leq \frac{z}{\beta_{1}}\right] & z \leq \beta_{1} \gamma_{0} \\ \operatorname{Prob}\left[\gamma \leq \gamma_{0}\right] & \beta_{1} \gamma_{0}<z \leq \beta_{2} \gamma_{0} \\ \operatorname{Prob}\left[\gamma \leq \frac{z}{\beta_{2}}\right] & z>\beta_{2} \gamma_{0}\end{cases}
$$

However, it should be noted that the power constraint is still in terms of $\alpha_{1}$ and $\alpha_{2}$ and is given by

$$
\alpha_{1} \operatorname{Prob}\left[\gamma \leq \gamma_{0}\right]+\alpha_{2}\left(1-\operatorname{Prob}\left[\gamma \leq \gamma_{0}\right]\right) \leq 1 .
$$

Using the above formulation, the minimization can be done similar to the method adopted in Section IV-B.2. The details are given in appendix C.

In summary, we have analyzed the outage performance of multiple antenna systems with imperfect channel information. In particular, we considered the following three cases: (i) preamble-based CSIR with no feedback, (ii) perfect CSIR with quantized feedback, and (iii) preamble-based CSIR with quantized feedback. We will present the numerical results in Section VII to illustrate the significant gains achievable using feedback.

\section{Outage Probability Bound with Erroneous Feedback}

The earlier analysis assumes that the feedback is received at the transmitter without any errors. Now, we will analyze the effect of any feedback error on quantizer and power control design. We will consider only the 1-bit feedback case to illustrate the effect of feedback error.

Let $P_{e}$ be the probability of error of the feedback bit. Let $\gamma \leq \gamma_{0}$ correspond to the quantizer bin for the 0 bit and $\gamma>\gamma_{0}$ correspond to the bin for the 1 bit. Let the power control allocate power $\alpha_{1} P$ when the received feedback bit is 0 and allocate power $\alpha_{2} P$ when the received feedback bit is 1 . Therefore, when there is no feedback error, the function $f(\gamma)$, which includes the effect of quantization and power control, will be

$$
f(\gamma)=\left\{\begin{array}{ll}
\alpha_{1} & \gamma \leq \gamma_{0} \\
\alpha_{2} & \gamma>\gamma_{0}
\end{array} .\right.
$$

When there is a feedback error, power $\alpha_{1} P$ will be allocated when $\gamma>\gamma_{0}$ and power $\alpha_{2} P$ 
will be allocated when $\gamma \leq \gamma_{0}$. Therefore, we define a new function $f_{e}(\gamma)$ given by

$$
f_{e}(\gamma)= \begin{cases}\alpha_{2} & \gamma \leq \gamma_{0} \\ \alpha_{1} & \gamma>\gamma_{0}\end{cases}
$$

to account for the quantization, feedback error and power control. Assuming that the feedback error event is independent of the particular realization of the forward channel fading coefficients $^{3}$, the overall outage probability can be upper bounded using a linear combination of the upper bounds for the case with and without an error event. This upper bound is given by

$$
\Pi_{\text {out }} \leq\left(1-P_{e}\right)\left[\operatorname{Prob}\left(f_{1}(\gamma) \gamma \leq\left(e^{R^{\prime}}-1\right)\right)\right]+P_{e}\left[\operatorname{Prob}\left(f_{1 e}(\gamma) \gamma \leq\left(e^{R^{\prime}}-1\right)\right)\right],
$$

where

$$
f_{1}(\gamma)=\frac{f(\gamma)}{\frac{M}{P_{d}}+\gamma_{p}^{2} f(\gamma)}
$$

and

$$
f_{1 e}(\gamma)=\frac{f_{e}(\gamma)}{\frac{M}{P_{d}}+\gamma_{p}^{2} f_{e}(\gamma)}
$$

For a given quantizer, this upper bound on outage probability is minimized numerically ${ }^{4}$ to obtain the optimal power control parameters $\alpha_{1}$ and $\alpha_{2}$. The quantizer threshold is chosen by searching over a pre-determined set. The numerical results are shown in Section VII.

\section{Vi. An Explicit Space-time Coding System with Feedback}

In the previous sections, we analyzed the effect of feedback on the outage probability of multiple transmit antenna systems. Outage probability is closely related to a practical performance measure, frame error rate. For large frame transmissions, the outage probability provides the achievable limit for the frame error rate. This is clear from the fact that when the frame length goes to infinity, an outage implies a frame error. Similarly, successful frame transmission is possible when there is no outage by the choice of a good coding scheme. Thus, for reasonably large frames, the outage probability acts like a lower bound on frame error rate performance and we can approach that performance by using good codes.

The space-time codes proposed already in the literature $[10,11]$ do not achieve the outage probability performance for perfect CSIR and no CSIT case. Therefore, the quantizer

${ }^{3}$ This can be achieved even when the reverse channel fading is dependent on the forward channel fading by using power control in the feedback channel to maintain a constant received power.

${ }^{4}$ using MATLAB 
and power control scheme need to be more conservative for a practical space-time code as compared to the optimal codes that achieves outage performance. In fact, direct use of the quantizer and power control schemes that minimize outage in conjunction with space-time codes can lead to higher frame error rates. This is because results based on mutual information are too optimistic about the code and might allocate too little power to overcome frame error even for good channel conditions. In order to determine the power control and quantizer for a practical code, we make use of the following simple but key observation. For full diversity codes, the average frame error rate has the same slope as the outage probability asymptotically as a function of the power constraint $P$ (in $\mathrm{dB})[11,20]$. In other words, the average frame error rate (without feedback) is equal to a modified outage probability that has a scaled power constraint. This can be written as

$$
F E R=\Pi_{\text {out }}\left(\frac{P}{k}\right)=\operatorname{Prob}\left(\log \left(1+\frac{P}{k M} \gamma\right)<R\right),
$$

where $k$ is the scaling. In order to obtain the quantizer and power control for a space-time code, we conjecture that the same relationship would hold in the presence of feedback. Thus, for each code, we would just need to use a different scaling factor depending on how far away the frame error rate performance of the code is from the non-feedback outage probability performance. This conjecture is supported by simulation results in Figure 10, where the modified outage with the scaled power is almost the same as the frame error rate.

Summarizing, we minimize the following modified outage probability

$$
\operatorname{Prob}\left(\log \left(1+\frac{P f(\gamma)}{k M} \gamma\right)<R\right)
$$

to obtain the quantizer and power control scheme. Thus, we still adopt a code-independent procedure for full diversity codes and apply it to individual codes by choosing the appropriate scaling $k$. In the case where we use a preamble, we use the appropriate modified outage function. The simulation results in Section VII show that the performance of the space-time code with this quantizer and power control scheme is similar to the frame error rate with this modified outage.

\section{Numerical Results and Discussion}

First, we will present the numerical results on the outage probability for the various preamble and feedback scenarios discussed in earlier sections. Then, we will illustrate the significance of these results in practical systems with space-time codes by demonstrating the 
gains from 1-bit feedback and power control for two codes, the simple Alamouti transformation that achieves maximum diversity [10] and the 16-state code from [11].

\section{A. Outage Probability Results}

In this section, we present the numerical results obtained by computing the outage probabilities and their upper bounds from the previous sections.

\section{A.1 1-bit Feedback}

Figures 3, 4, and 5 show the performance gains from feedback for multiple antenna systems using a frame length $(T)$ of 1000 . Figure 3 plots outage probability versus transmission rate $R$ for a 4 transmit antenna, 1 receive antenna system at an average power constraint $P$ of $14 d B$. It shows the increase in achievable rate using feedback for a fixed outage probability considering the effect of preamble and quantization in feedback. Figure 4 plots outage probability versus number of transmit antennas for transmission rate $R=2 \mathrm{bits} / \mathrm{s} / \mathrm{Hz}$ and average power constraint $P=14 d B$. This plot shows the increasing gains from feedback as the number of transmit antennas increases. Figure 5 plots outage probability versus $P$ for a 4 transmit antenna system at $R=2 \mathrm{bits} / \mathrm{s} / \mathrm{Hz}$. This plot shows the significant gain from only one bit of feedback. The performance of a preamble-based scheme with feedback is significantly better than a scheme no feedback and perfect channel information at the receiver. For example, at an outage probability of $10^{-4}$ we have a gain of more than $5 d B$ in a preamble-based system for 1-bit feedback compared to a system with perfect CSIR. Furthermore, the outage decreases at a much faster rate with $P$ for the 1-bit feedback case than for the perfect CSIR case. The outage with perfect CSIR has a slope equal to the number of transmit antennas or the diversity. In all these results, the training power per symbol was chosen to be the best value from the set $\{P, 2 P, \ldots, 10 P\}$. A maximum training power constraint can be easily enforced by restricting this search set for training power. Significant performance gains from feedback were observed even when the maximum training power per symbol was restricted to $3 P$.

Figure 6 shows the effect of varying the frame length on the outage performance. Using a larger frame allows more power to be used for training without taking away too much from data transmission. As the frame length increases, the fraction of time spent in the preamble goes to zero. Therefore, as $T$ increases, performance approaches the performance with perfect CSIR and 1-bit feedback. 
Unlike the 1-bit feedback case where we optimize for the best quantizer, for the multiple bit feedback case we will choose from a fixed search set of quantizers and use the optimal power control from Section IV-C.1. Figure 7 shows the negligible difference in the outage bounds for 1-bit feedback obtained in the following two methods:

1. Obtain optimal quantizer and power control using results in Section IV-C.2.

2. Search over fixed set of quantizers and use optimal power control scheme for a fixed quantizer from Section IV-C.1.

The thresholds over which the search was performed were such that the probability of $\gamma$ being less than the threshold was from $\{0.001,0.002, \ldots, 0.009,0.01,0.02, \ldots, 0.09,0.1,0.2$, ..., 0.9$\}$.

\section{A.2 2-bit Feedback}

Figure 8 shows the outage performance versus $P$ for the two bit feedback case for a 4 transmit antenna system at $R=2 \mathrm{bits} / \mathrm{s} / \mathrm{Hz}$ and $T=1000$. There is still a significant additional gain of about $2 d B$ at $10^{-4}$ from the second bit of feedback although the marginal gain from the second bit of feedback is lower as compared to the gain from the first bit of feedback. In this case, the three thresholds $\gamma_{1}, \gamma_{2}$ and $\gamma_{3}$, required to specify the quantizer were obtained by a search. All quantizers with $\gamma_{1} \leq \gamma_{2} \leq \gamma_{3}$ and $\gamma_{i}$ such that probability of $\gamma \leq \gamma_{i}$ belongs to $\{0.001,0.002, \ldots, 0.009,0.01,0.02, \ldots, 0.090 .1,0.2, \ldots, 0.9\}$ were used in the search set.

\section{A.3 Erroneous Feedback}

Figure 9 shows the outage performance versus $P$ for a 4 transmit antenna, 1 receive antenna system with $R=2 \mathrm{bits} / \mathrm{s} / \mathrm{Hz}, T=1000$ in the presence of feedback error. The following 3 feedback error rates are considered: (i) 0.01 (ii) 0.001 (iii) bit error rate (as a function of $P$ ) of a binary noncoherent signaling with 4 channels with power control such that total received power is $P$ (equation 12-1-13 from [21]). Although there is degradation in performance compared to the no error case, the overall system is still much better than a system with no feedback.

\section{B. Frame Error Rates for Practical Codes}

In this section, we show the simulation results for 2 transmit antenna, 1 receive antenna systems with preamble and 1-bit feedback. The power control scheme and quantizer are 
determined using the modified outage function discussed in Section VI.

For the Alamouti scheme [10], we use a scaling $k=3.2$ in the modified outage function. This scaling factor is obtained based on the performance difference between the Alamouti scheme and outage. The quantizer is obtained by search and the optimal power control is found using Section IV-C.1. Figure 10 shows the frame error rate performance of a preamble based scheme with and without the 1-bit feedback. Significant performance gain can be observed by the use of feedback and power control. For example, there is a gain of about 2 $d B$ from 1-bit feedback at a frame error rate of 0.02 . Furthermore, the frame error rate of the system with feedback is also compared with the minimum modified outage probability to show that they are almost the same. This supports the use of the modified outage function in Section VI to describe the average frame error rate even in the presence of 1-bit feedback.

In the outage analysis, the coding rate is increased in the feedback case to overcome the loss of one bit for feedback. However, it is difficult to change the rate of code arbitrarily in practice. Here, we fix the coding rate and thereby suffer a small loss in rate $(1 /(T+1))$. We account for this in the simulation results by adjusting the $P$ axis for the no feedback case. We shift the no feedback curve to the left corresponding the gain that would have been achieved in the no feedback case with the lowered rate of transmission. This adjustment is motivated by the normalized SNR used in $[22,23]$ to compare of codes of different rates.

Finally, Figure 11 shows the performance gain from feedback and power control in terms of frame error rate for the 16-state space-time code in [11] using QPSK symbols. There is approximately $2.5 \mathrm{~dB}$ gain from feedback at a frame error rate of $10^{-2}$. In this case, the appropriate scaling factor is found to be 2.3, which is lower than for the Alamouti scheme as expected. The scaling factor is lower because this code is stronger and therefore closer to outage performance than the Alamouti scheme.

\section{Conclusions}

In this paper, we have evaluated the gains in outage performance from feedback for multiple antenna systems considering the following practical issues in our analysis and design: (i) channel information is imperfect both at the receiver and at the transmitter, and (ii) channel estimation and feedback schemes consume part of the total available resources for the system. Our results show that significant performance gains can be achieved even with one bit of feedback. For example, a 4 transmit antenna system over a block fading channel of block length 1000 can achieve a $5 d B$ gain from 1-bit feedback at an outage probability of $10^{-4}$. 
The performance improvements can be achieved by the implementation of power control at the transmitter based on the quantized feedback of the instantaneous channel signal to noise ratio. The upper bounds on outage probability performance are minimized analytically for the 1-bit feedback case to obtain the optimal quantizer and power control strategy. For the multiple bit feedback case, we determine the optimal power control strategy for a fixed quantizer. Finally, we demonstrate the significant gains achievable in frame error rate performance of practical space-time code systems with power control and 1-bit feedback. We design power control and 1-bit feedback for space-time codes to achieve more than $2 d B$ gain at frame error rates of 0.01 .

\section{APPENDiX}

\section{Obtaining the LOWER Bound In (22)}

The details of obtaining (22) from (21) by substituting $\mathbf{g}$ are as follows. Since $\mathbf{g}$ is the MMSE estimator, we have $\mathbf{g} E\left[y y^{*} \mid \hat{\mathbf{h}}\right]=E\left[\mathbf{x} y^{H} \mid \hat{\mathbf{h}}\right]$. Therefore, we have

$$
\begin{aligned}
& E\left[(\mathbf{x}-\mathbf{g} y)(\mathbf{x}-\mathbf{g} y)^{H} \mid \hat{\mathbf{h}}\right]=E\left[\mathbf{x x ^ { H }}\right]-E\left[\mathbf{x} y^{H} \mathbf{g}^{H} \mid \hat{\mathbf{h}}\right] \\
& +E\left[\mathbf{g} y y^{H} \mathbf{g}^{H} \mid \hat{\mathbf{h}}\right]-E\left[\mathbf{g} y \mathbf{x}^{H} \mid \hat{\mathbf{h}}\right] \\
& =E\left[\mathbf{x x}^{H}\right]-E\left[\mathbf{g} y \mathbf{x}^{H} \mid \hat{\mathbf{h}}\right] \\
& =Q-E\left[\frac{\frac{P_{d}}{M} \hat{\mathbf{h}}^{H}(\hat{\mathbf{h}} \mathbf{x}+\hat{w}) \mathbf{x}^{H}}{\frac{P_{d}}{M} \hat{\mathbf{h}}^{H}+E\left[|\hat{w}|^{2} \mid \hat{\mathbf{h}}=\hat{\mathbf{h}}\right]} \mid \hat{\mathbf{h}}\right] .
\end{aligned}
$$

Using (18), we get

$$
E\left[(\mathbf{x}-\mathbf{g} y)(\mathbf{x}-\mathbf{g} y)^{H} \mid \hat{\mathbf{h}}\right]=Q\left(I-\frac{\frac{P_{d}}{M} \hat{\mathbf{h}}^{H} \hat{\mathbf{h}}}{\frac{P_{d}}{M} \hat{\mathbf{h}}^{H}+E\left[|\hat{w}|^{2} \mid \hat{\mathbf{h}}\right]}\right) .
$$

Substituting this back in (21), we have

$$
I(\mathbf{x} ; y \mid \hat{\mathbf{h}}) \geq \frac{T-M}{M}\left[\log (\operatorname{det}(\pi e Q))-\log \left(\operatorname{det}\left(\pi e Q\left(I-\frac{\frac{P_{d}}{M} \hat{\mathbf{h}}^{H} \hat{\mathbf{h}}}{\frac{P_{d}}{M} \hat{\mathbf{h}}^{H}+E\left[|\hat{w}|^{2} \mid \hat{\mathbf{h}}\right]}\right)\right)\right)\right] .
$$

Using $\operatorname{det}(A B)=(\operatorname{det} A)(\operatorname{det} B)$, we get

$$
I(\mathbf{x} ; y \mid \hat{\mathbf{h}}) \geq-\frac{T-M}{M} \log \left(\operatorname{det}\left(I-\frac{\frac{P_{d}}{M} \hat{\mathbf{h}}^{H} \hat{\mathbf{h}}}{\frac{P_{d}}{M} \hat{\mathbf{h}}^{H}+E\left[|\hat{w}|^{2} \mid \hat{\mathbf{h}}=\hat{\mathbf{h}}\right]}\right)\right) .
$$

Using $\operatorname{det}(I+A B)=\operatorname{det}(I+B A)[1]$, we get

$$
I(\mathbf{x} ; y \mid \hat{\mathbf{h}}) \geq-\frac{T-M}{M} \log \left(\operatorname{det}\left(1-\frac{\frac{P_{d}}{M} \hat{\mathbf{h}}^{H}}{\frac{P_{d}}{M} \hat{\mathbf{h}}^{H}+E\left[|\hat{w}|^{2} \mid \hat{\mathbf{h}}\right]}\right)\right)=\frac{T-M}{M} \log \left(1+\frac{\frac{P_{d}}{M} \hat{\mathbf{h}} \hat{\mathbf{h}}^{H}}{E\left[|\hat{w}|^{2} \mid \hat{\mathbf{h}}\right]}\right) .
$$


From (16), we have

$$
E\left[|\hat{w}|^{2} \mid \hat{\mathbf{h}}\right]=1+\gamma_{p}^{2} P_{d} .
$$

Thus, we get the required lower bound in (22).

$$
I(\mathbf{x} ; y \mid \hat{\mathbf{h}}) \geq=\frac{T-M}{M} \log \left(1+\frac{P_{d} \hat{\mathbf{h}} \hat{\mathbf{h}}^{H}}{M\left(1+\gamma_{p}^{2} P_{d}\right)}\right) .
$$

\section{Perfect CSIR with 1-Bit Feedback}

Case 1: $\alpha_{1}>\alpha_{2}$

We need to minimize $P\left[\gamma \leq z / \alpha_{1}\right]$ under the constraints $z \leq \alpha_{2} \gamma_{0}$ and $\alpha_{1} \operatorname{Prob}\left[\gamma \leq \gamma_{0}\right]+$ $\alpha_{2}\left(1-\operatorname{Prob}\left[\gamma \leq \gamma_{0}\right]\right) \leq 1$. Since $\mathbf{h}$ is distributed as $\mathcal{C N}(\mathbf{0}, \mathbf{I})$, we have

$$
\operatorname{Prob}[\gamma \leq a]=1-e^{-a} \sum_{k=0}^{M-1} \frac{a^{k}}{k !} .
$$

Using this expression for the cumulative distribution function of $\gamma$ and the constraints, we construct the function, $\mathrm{g}\left(\alpha_{1}, \alpha_{2}, \gamma_{0}, \lambda_{1}, \lambda_{2}\right)$, as

$$
\begin{aligned}
g\left(\alpha_{1}, \alpha_{2}, \gamma_{0}, \lambda_{1}, \lambda_{2}\right)= & 1-e^{-\frac{z}{\alpha_{1}}} \sum_{k=0}^{M-1} \frac{\left(z / \alpha_{1}\right)^{k}}{k !}+\lambda_{1}\left[z-\alpha_{2} \gamma_{0}\right] \\
& +\lambda_{2}\left[\left(\alpha_{1}-\alpha_{2}\right)\left(1-e^{-\gamma_{0}} \sum_{k=0}^{M-1} \frac{\gamma_{0} k}{k !}\right)+\alpha_{2}-1\right],
\end{aligned}
$$

where $\lambda_{1}$ and $\lambda_{2}$ are Lagrange multipliers. We now solve for the variables $\alpha_{1}, \alpha_{2}, \gamma_{0}, \lambda_{1}$ and $\lambda_{2}$ by setting the partial derivatives of $g$ with respect to each of these variables to zero. The unconstrained minimization of the function $g$ gives us the parameters that minimize outage probability under the constraints described earlier.

The partial derivatives with respect to $\alpha_{1}, \alpha_{2}, \gamma_{0}, \lambda_{1}$ and $\lambda_{2}$ are given below.

$$
\begin{gathered}
\frac{\partial g}{\partial \alpha_{1}}=e^{-\frac{z}{\alpha_{1}}} \frac{\left(z / \alpha_{1}\right)^{M-1}}{(M-1) !}\left(\frac{-z}{\alpha_{1}^{2}}\right)+\lambda_{2}\left[1-e^{-\gamma_{0}} \sum_{k=0}^{M-1} \frac{\gamma_{0}^{k}}{k !}\right]=0 \\
\frac{\partial g}{\partial \alpha_{2}}=-\lambda_{1} \gamma_{0}-\lambda_{2}\left[1-e^{-\gamma_{0}} \sum_{k=0}^{M-1} \frac{\gamma_{0}^{k}}{k !}\right]+\lambda_{2}=0 \\
\frac{\partial g}{\partial \gamma_{0}}=-\lambda_{1} \alpha_{2}+\lambda_{2}\left(\alpha_{1}-\alpha_{2}\right) e^{-\gamma_{0}} \frac{\gamma_{0}{ }^{M-1}}{(M-1) !}=0 \\
\frac{\partial g}{\partial \lambda_{1}}=z-\alpha_{2} \gamma_{0}=0
\end{gathered}
$$




$$
\frac{\partial g}{\partial \lambda_{2}}=\left(\alpha_{1}-\alpha_{2}\right)\left(1-e^{-\gamma_{0}} \sum_{k=0}^{M-1} \frac{\gamma_{0}^{k}}{k !}\right)+\alpha_{2}-1=0
$$

Substituting for $\lambda_{1}$ in (52) in terms of $\lambda_{2}$ from (53), and eliminating $\lambda_{2}$ from the resulting (since it appears as a constant), we get

$$
-\frac{\left(\alpha_{1}-\alpha_{2}\right)}{\alpha_{2}} e^{-\gamma_{0}} \frac{\gamma_{0}^{M-1}}{(M-1) !} \gamma_{0}-\left[1-e^{-\gamma_{0}} \sum_{k=0}^{M-1} \frac{\gamma_{0}^{k}}{k !}\right]+1=0 .
$$

Now, substituting $\gamma_{0}$ by $z / \alpha_{2}$ (from (54)), in equations (56) and (55), we get

$$
\begin{gathered}
-\frac{\left(\alpha_{1}-\alpha_{2}\right)}{\alpha_{2}} e^{-\frac{z}{\alpha_{2}}} \frac{\left(z / \alpha_{2}\right)^{M-1}}{(M-1) !} \frac{z}{\alpha_{2}}-\left[1-e^{-\frac{z}{\alpha_{2}}} \sum_{k=0}^{M-1} \frac{\left(z / \alpha_{2}\right)^{k}}{k !}\right]+1=0 . \\
\left(\alpha_{1}-\alpha_{2}\right)\left(1-e^{-\frac{z}{\alpha_{2}}} \sum_{k=0}^{M-1} \frac{\left(z / \alpha_{2}\right)^{k}}{k !}\right)+\alpha_{2}-1=0
\end{gathered}
$$

Now, we have reduced the problem to solving for two variables, $\alpha_{1}$ and $\alpha_{2}$, from the two equations (57) and (58). Eliminating $\alpha_{1}$ from these equations, we get a single equation in $\alpha_{2}$,

$$
\left(\alpha_{2}-1\right) \frac{z}{\alpha_{2}^{2}} \frac{\left(z / \alpha_{2}\right)^{M-1}}{(M-1) !}+\left(\sum_{k=0}^{M-1} \frac{\left(z / \alpha_{2}\right)^{k}}{k !}\right)\left(1-e^{-z / \alpha_{2}} \sum_{k=0}^{M-1} \frac{\left(z / \alpha_{2}\right)^{k}}{k !}\right)=0 .
$$

After solving for $\alpha_{2}$ from (59), we can get $\alpha_{1}$ as

$$
\alpha_{1}=\frac{1-\alpha_{2}}{1-e^{-z / \alpha_{2}} \sum_{k=0}^{M-1} \frac{\left(z / \alpha_{2}\right)^{k}}{k !}}+\alpha_{2}
$$

and $\gamma_{0}$ as $z / \alpha_{2}$. Thus, the minimum outage probability is given as

$$
\Pi_{\text {out }}=\operatorname{Prob}\left[\gamma \leq \frac{z}{\alpha_{1}}\right]
$$

Case 2: $\alpha_{1}=0$

We need to solve the equations $\alpha_{2} \gamma_{0}=z$ and $\alpha_{2}\left(1-\operatorname{Prob}\left[\gamma \leq \gamma_{0}\right]\right)=1$ to obtain $\alpha_{2}$ and $\gamma_{0}$. Therefore, $\alpha_{2}$ is obtained by solving

$$
\alpha_{2}\left[e^{-\frac{z}{\alpha_{2}}} \sum_{k=0}^{M-1} \frac{\left(z / \alpha_{2}\right)^{k}}{k !}\right]=1
$$

and $\gamma_{0}$ is obtained as $z / \alpha_{2}$. The minimum outage probability is Prob $\left[\gamma \leq \gamma_{0}\right]$. In Section VII, (62) is solved numerically ${ }^{5}$.

\footnotetext{
${ }^{5}$ using the fzero command in MATLAB.
} 


\section{Preamble-BAsed CSIR With 1-Bit FEedBack}

Case 1: $\alpha_{1}>\alpha_{2}$

We need to minimize $P\left[\gamma \leq z / \beta_{1}\right]$ under the constraints $z \leq \beta_{2} \gamma_{0}$ and $\alpha_{1} \operatorname{Prob}\left[\gamma \leq \gamma_{0}\right]+$ $\alpha_{2}\left(1-\operatorname{Prob}\left[\gamma \leq \gamma_{0}\right]\right) \leq 1$. Since $\gamma=\hat{\mathbf{h}} \hat{\mathbf{h}}^{H}$, we have

$$
\operatorname{Prob}[\gamma \leq a]=1-e^{-a / s} \sum_{k=0}^{M-1} \frac{(a / s)^{k}}{k !}
$$

where

$$
s=\frac{P_{t} / M}{P_{t} / M+1}
$$

Using this expression for the cumulative distribution function of $\gamma$ and the constraints, we construct the function, $\mathrm{g}\left(\alpha_{1}, \alpha_{2}, \gamma_{0}, \lambda_{1}, \lambda_{2}\right)$, as

$$
\begin{aligned}
g\left(\alpha_{1}, \alpha_{2}, \gamma_{0}, \lambda_{1}, \lambda_{2}\right)= & 1-e^{-\frac{z}{\beta_{1} s}} \sum_{k=0}^{M-1} \frac{\left(z / \beta_{1} s\right)^{k}}{k !}+\lambda_{1}\left[z-\beta_{2} \gamma_{0}\right] \\
+ & \lambda_{2}\left[\left(\alpha_{1}-\alpha_{2}\right)\left(1-e^{-\gamma_{0} / s} \sum_{k=0}^{M-1} \frac{\left(\gamma_{0} / s\right)^{k}}{k !}\right)+\alpha_{2}-1\right],
\end{aligned}
$$

where $\lambda_{1}$ and $\lambda_{2}$ are Lagrange multipliers. We now solve for the variables $\alpha_{1}, \alpha_{2}, \gamma_{0}, \lambda_{1}$ and $\lambda_{2}$ by setting the partial derivatives of $g$ with respect to each of these variables to zero. The unconstrained minimization of the function $g$ gives us the parameters that minimize the upper bound on outage probability under the constraints described earlier.

Following similar steps as in appendix $\mathrm{B}$, we get the following equation in $\alpha_{2}$.

$$
\frac{\left(\alpha_{2}-1\right)}{\beta_{2} s} \frac{\left(z / \beta_{2} s\right)^{M-1}}{(M-1) !} \frac{\left(z / \beta_{2}\right) M}{P_{d}\left(\frac{M}{P_{d}}+\gamma_{p}^{2} \alpha_{2}\right)^{2}}+\left(\sum_{k=0}^{M-1} \frac{\left(z / \beta_{2} s\right)^{k}}{k !}\right)\left(1-e^{-z / \beta_{2} s} \sum_{k=0}^{M-1} \frac{\left(z / \beta_{2} s\right)^{k}}{k !}\right)=0
$$

where

$$
\beta_{2}=\frac{\alpha_{2}}{\frac{M}{P_{d}}+\gamma_{p}^{2} \alpha_{2}}
$$

Once we solve for $\alpha_{2}, \alpha_{1}$ is obtained as

$$
\alpha_{1}=\frac{1-\alpha_{2}}{1-e^{-z / \beta_{2} s} \sum_{k=0}^{M-1} \frac{\left(z / \beta_{2} s\right)^{k}}{k !}}+\alpha_{2},
$$

and $\gamma_{0}$ is obtained as $z / \beta_{2}$. The minimum upper bound on outage probability is

$$
\Pi_{\text {out }}=\operatorname{Prob}\left[\gamma \leq \frac{z}{\beta_{1}}\right]
$$


Case 2: $\alpha_{1}=0$

We need to solve the equations $\beta_{2} \gamma_{0}=z$ and $\alpha_{2}\left(1-\operatorname{Prob}\left[\gamma \leq \gamma_{0}\right]\right)=1$ to obtain $\alpha_{2}$ and $\gamma_{0}$. Therefore, $\alpha_{2}$ is obtained by solving

$$
\alpha_{2}\left[e^{-\frac{z}{\beta_{2}} s} \sum_{k=0}^{M-1} \frac{\left(z / \beta_{2} s\right)^{k}}{k !}\right]=1,
$$

and $\gamma_{0}$ is obtained as $z / \beta_{2}$. The minimum outage probability is Prob $\left[\gamma \leq \gamma_{0}\right]$.

\section{REFERENCES}

[1] E. Telatar, "Capacity of multi-antenna gaussian channels," AT \& T Bell Labs Internal Tech. Memo, June 1995.

[2] Thomas L.Marzetta and Bertrand M.Hochwald, "Capacity of a mobile multiple-antenna communication link in Rayleigh flat fading," IEEE Transactions on Information Theory, vol. 45, no. 1, pp. 139-157, Jan 1999.

[3] E. Biglieri, G. Caire, and G. Taricco, "Limiting performance of block-fading channels with multiple antennas," submitted to IEEE Transactions on Information Theory, August 1999.

[4] A. Narula, M. D. Trott, and G. W. Wornell, "Performance limits of coded diversity methods for transmitter antenna arrays," IEEE Transactions on Information Theory, vol. 45, no. 7, pp. 2418-2433, Nov 1999.

[5] A. J. Goldsmith and P. P. Varaiya, "Capacity of fading channels with channel side information," IEEE Transactions on Information Theory, vol. 43, no. 6, pp. 1986-92, Nov 1997.

[6] C. Kose and D. L. Goeckel, "On power adaptation in adaptive signaling systems," IEEE Transactions on Communications, vol. 48, no. 11, pp. 1769-1773, Nov 2000.

[7] M. Medard, "The effect upon channel capacity in wireless communications of perfect and imperfect knowledge of the channel," IEEE Transactions on Information Theory, vol. 46, no. 3, pp. 933-946, May 2000.

[8] E. Visotsky and U. Madhow, "Space-time precoding with imperfect feedback," in Proceedings ISIT 2000, Sorrento, Italy, June 2000.

[9] A. Sabharwal, E. Erkip, and B. Aazhang, "On channel state information in multiple antenna block fading channels," in Proceedings ISITA, Hawaii, Nov 2000.

[10] S. M. Alamouti, "A simple transmit diversity technique for wireless communications," IEEE Journal on Selected Areas in Communications, vol. 16, no. 8, pp. 1451-1458, Oct 1998.

[11] V. Tarokh, N. Seshadri, and A. R. Calderbank, "Space-time codes for high data rate wireless communication: performance criterion and code construction," IEEE Transactions on Information Theory, vol. 44, no. 2, pp. 744-765, Mar 1998.

[12] H. Viswanathan, "Capacity of markov channels with receiver CSI and delayed feedback," IEEE Transactions on Information Theory, vol. 45, no. 2, pp. 761-771, Mar 1999.

[13] G. Caire, G. Taricco, and E. Biglieri, "Optimum power control over fading channels," IEEE Transactions on Information Theory, vol. 45, no. 5, pp. 1468-1489, Jul 1999.

[14] E. Biglieri, G. Caire, and G. Taricco, "Limiting performance of block-fading channels with multiple antennas," Submitted to IEEE Transactions on Information Theory, Sep 1999.

[15] A. Narula, M. J. Lopez, M. D. Trott, and G. W. Wornell, "Efficient use of side information in multiple-antenna data transmission over fading channels," IEEE Journal on Selected Areas of Communications, vol. 16, no. 8, pp. 1423-1436, Oct 1998.

[16] S. A. Jafar and A. Goldsmith, "On optimality of beamforming for multiple antenna systems with imperfect feedback," in Proceedings ISIT 2001, Washington D. C., USA, June 2001. 
[17] K. K. Mukkavilli, A. Sabharwal, M. Orchard, and B. Aazhang, "Transmit diversity with channel feedback," in Proceedings International Symposium on Telecommunications, Tehran, Iran, September 2001.

[18] H. V. Poor, An Introduction to Signal Detection and Estimation, Springer-Verlag, 1994.

[19] A. Lapidoth and S. Shamai, "Fading channels: How perfect need "perfect side information" be?," in Proceedings IEEE Workshop on Information Theory, Kruger National Park, South Africa, June 1999.

[20] T. Muharemovic and B. Aazhang, "Information theoretic optimality of orthogonal space time transmission schemes and concatenated code construction," in Proceedings International Conference on Telecommunications, May 2000.

[21] J. G. Proakis, Digital Communications, McGraw Hill, 1995.

[22] G. D. Forney and L-F. Wei, "Multidimensional constellations - part I: Introduction, figures of merit, and generalized cross constellations," IEEE Journal on Selected Areas in Communications, vol. 7, no. 6, pp. 877892, Aug 1989.

[23] M. V. Eyuboglu and G. D. Forney, "Lattice and trellis quantization with lattice- and trellis-bounded codebooks - high-rate theory for memoryless sources," IEEE Transactions on Information Theory, vol. 39, no. 1, pp. 46-59, Jan 1993. 


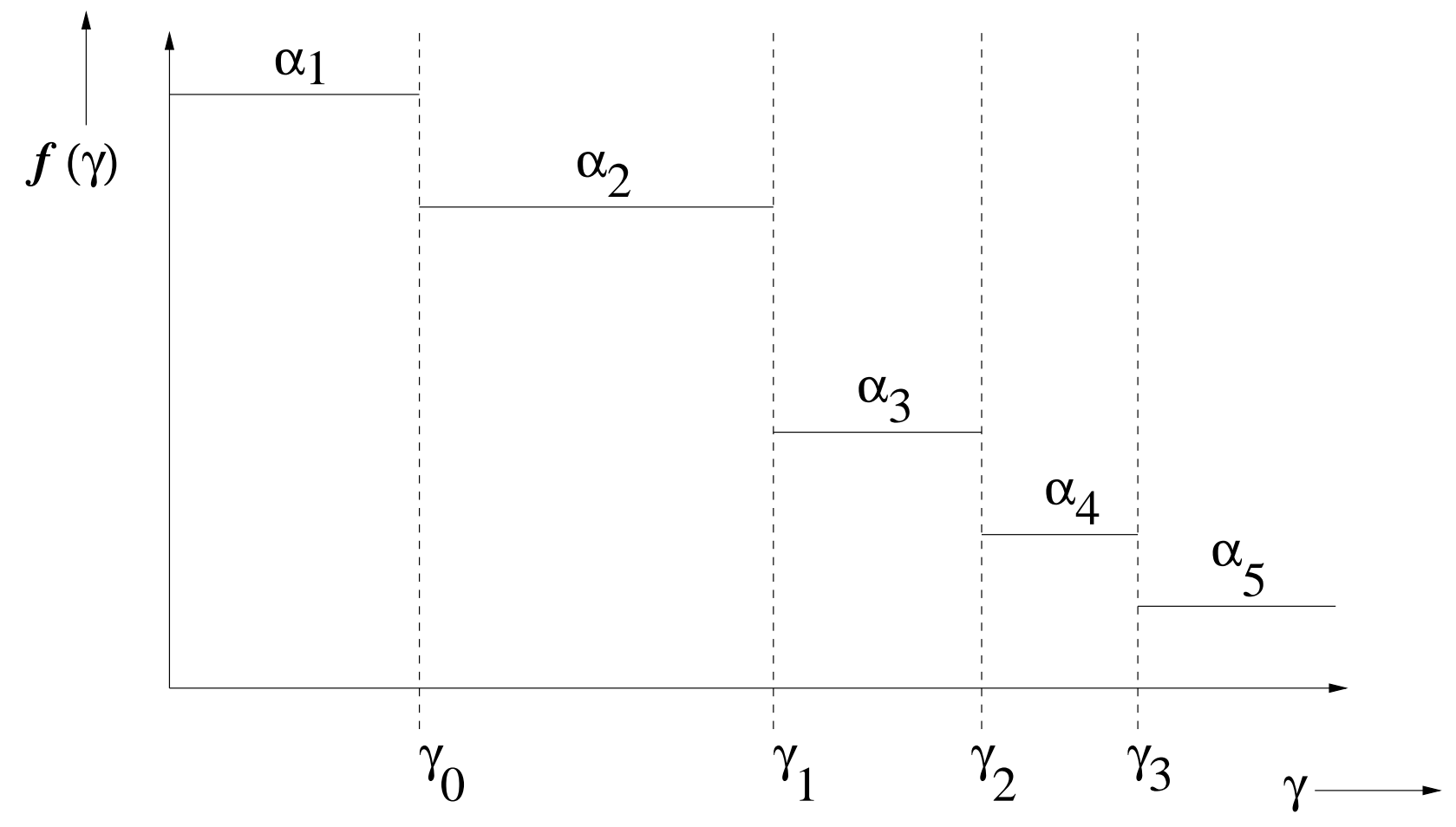

Fig. 1. Power control function 


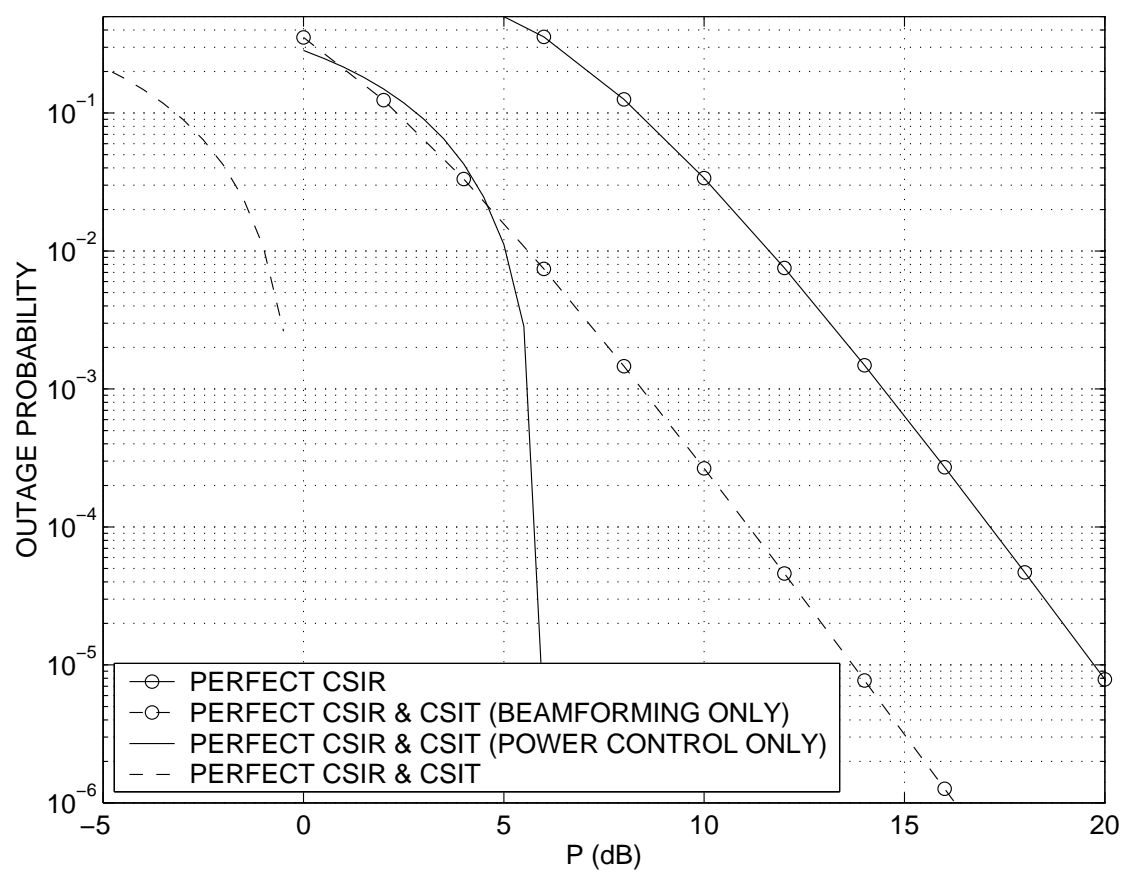

Fig. 2. Performance gains from feedback - outage probability versus average power constraint for rate $R=2 \mathrm{bits} / \mathrm{s} / \mathrm{Hz}, M=4$ transmit antennas, frame length $T=1000$. For the Perfect CSIR \& CSIT curve, outage is zero for $P$ larger than the plotted range. 


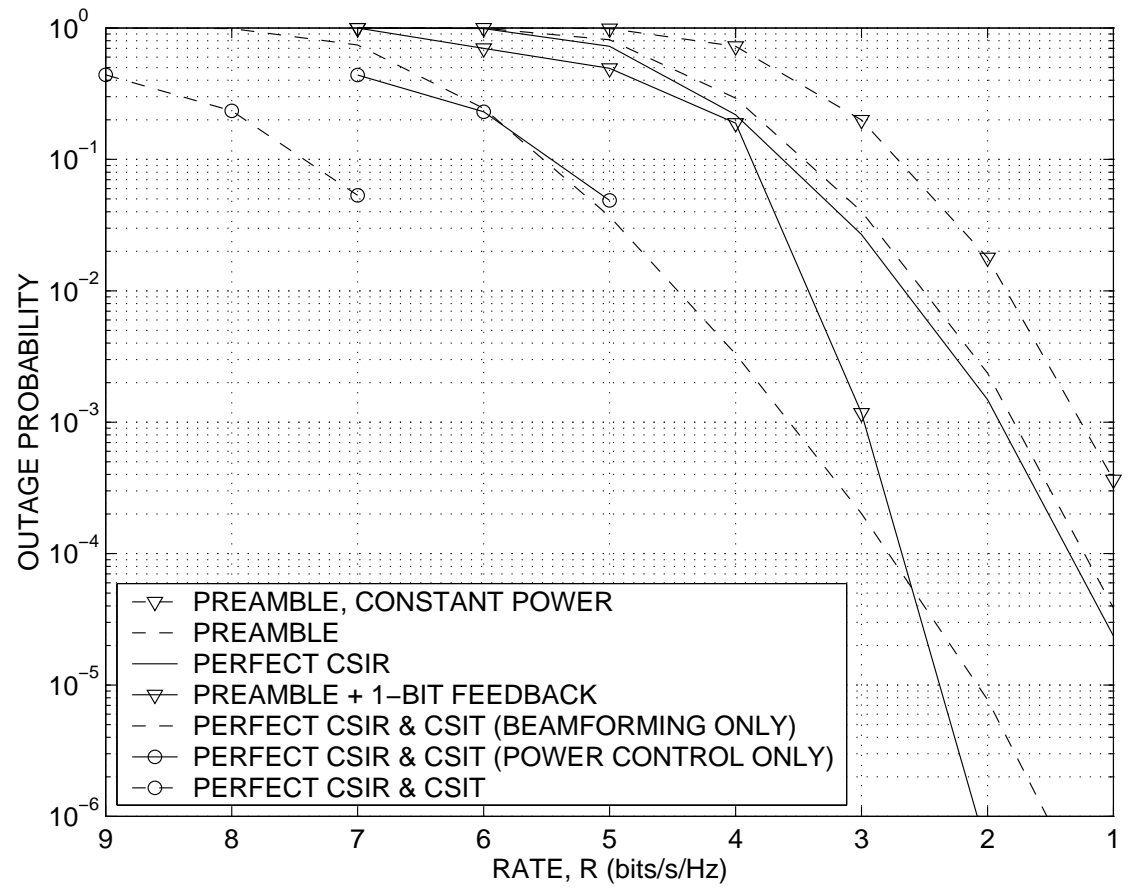

Fig. 3. Outage probability versus transmission rate for $M=4$ transmit antennas, average power constraint $P=14 d B$, frame length $T=1000$. For the Perfect CSIR \& CSIT and the Perfect CSIR \& CSIT (Power control only) curves, outage is zero for $R$ smaller than the plotted range. 


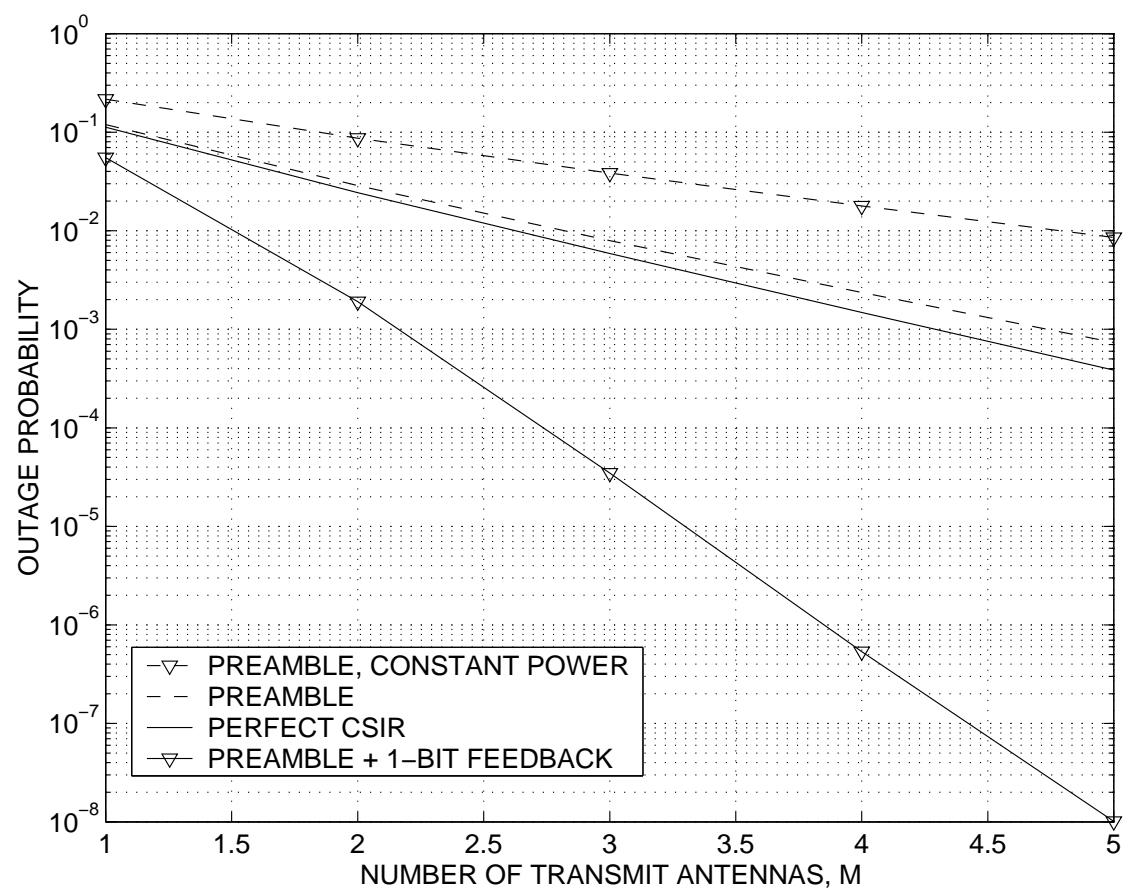

Fig. 4. Outage probability versus number of transmit antennas for rate $R=2 \mathrm{bits} / \mathrm{s} / \mathrm{Hz}$, average power constraint $P=14 d B$, frame length $T=1000$.

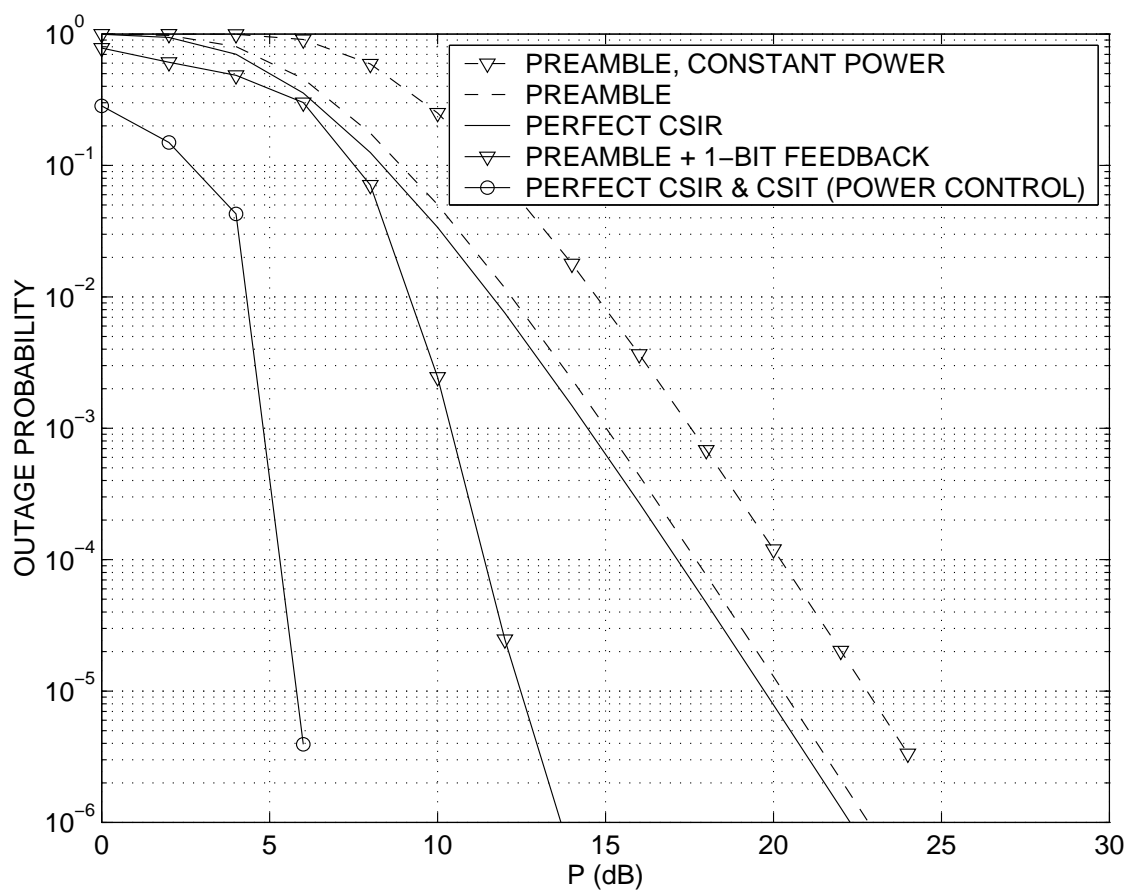

Fig. 5. Performance gains from 1-bit feedback - outage probability versus average power constraint for rate $R=2 \mathrm{bits} / \mathrm{s} / \mathrm{Hz}, M=4$ transmit antennas, frame length $T=1000$. 


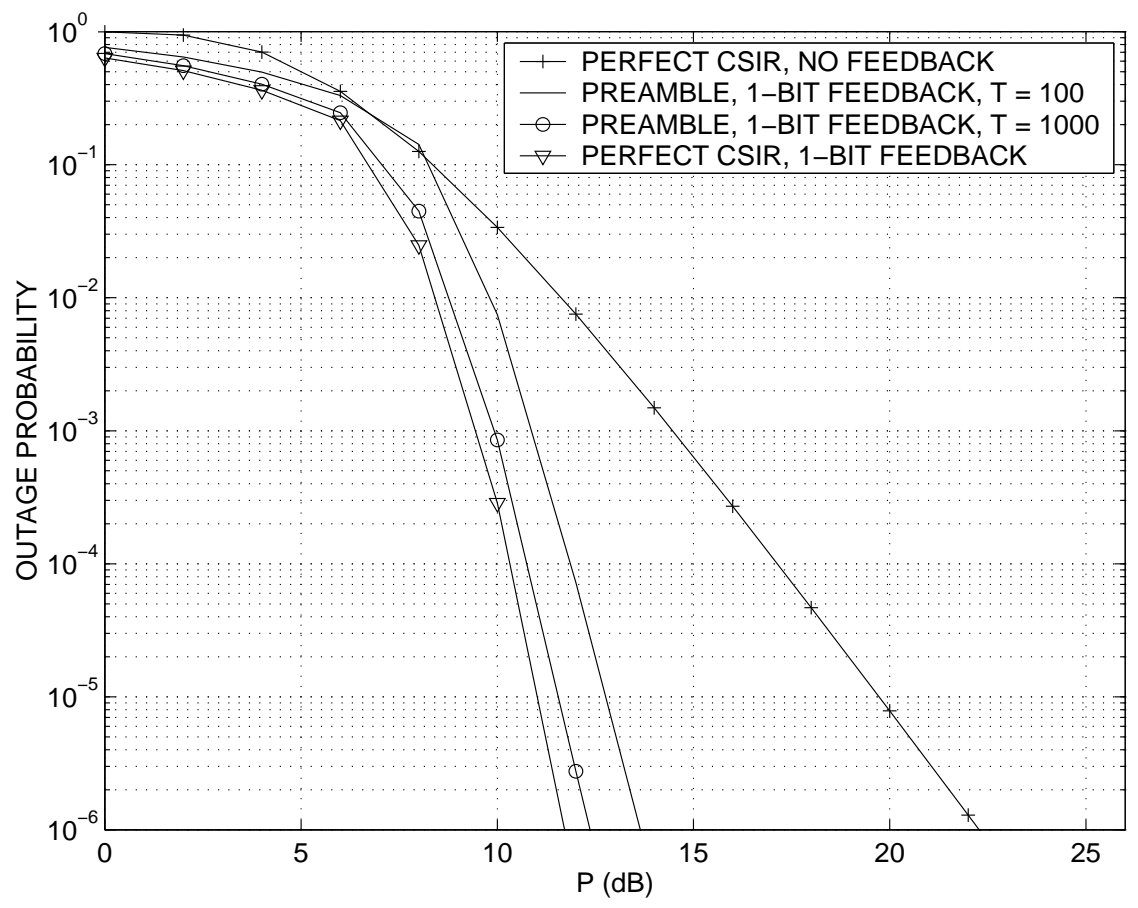

Fig. 6. Effect of frame length $(T)$ on outage probability - outage probability versus power constraint for $M=4$ transmit antennas, rate $R=2 \mathrm{bits} / \mathrm{s} / \mathrm{Hz}$. 


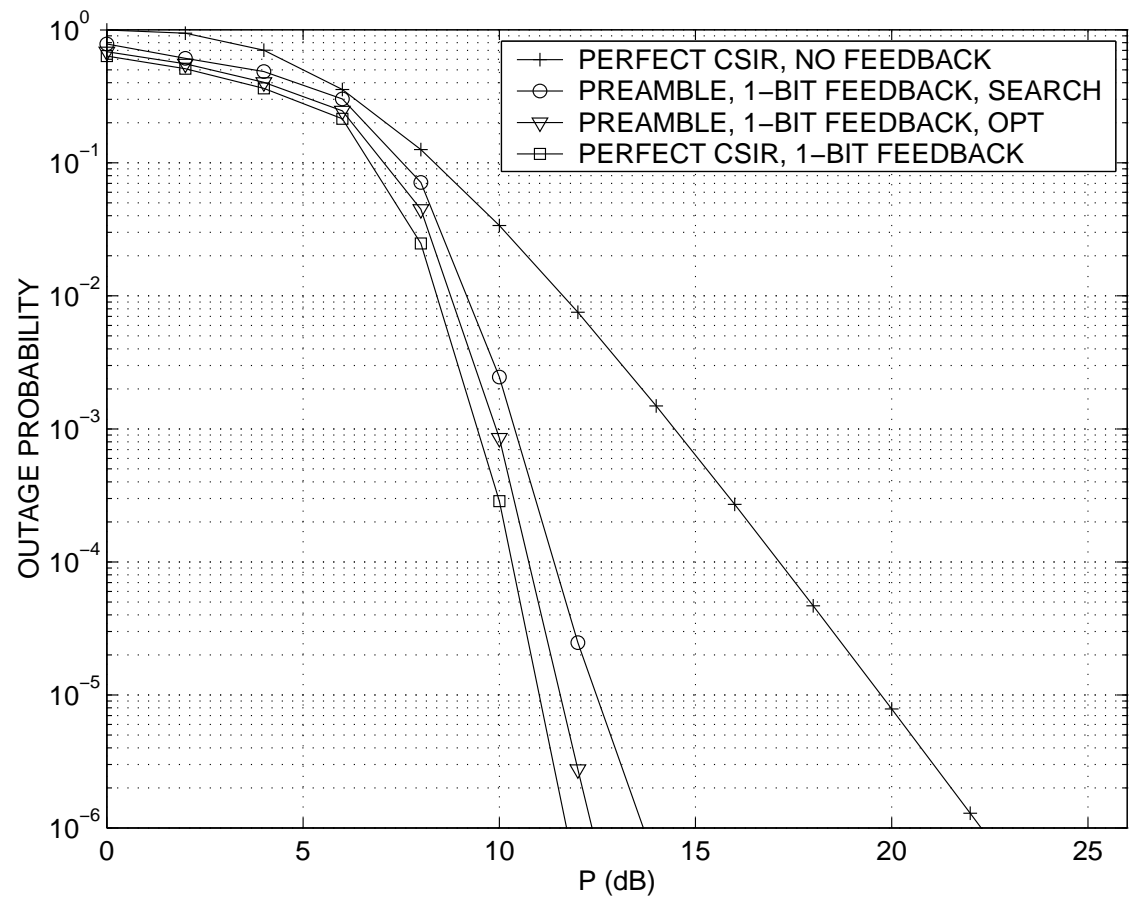

Fig. 7. Comparison of the two upper bounds obtained by (i) quantizer search and (ii) analytical optimization - outage probability versus power constraint for $M=4$ transmit antennas, frame length $T=1000$, rate $R=2 \mathrm{bits} / \mathrm{s} / \mathrm{Hz}$. 


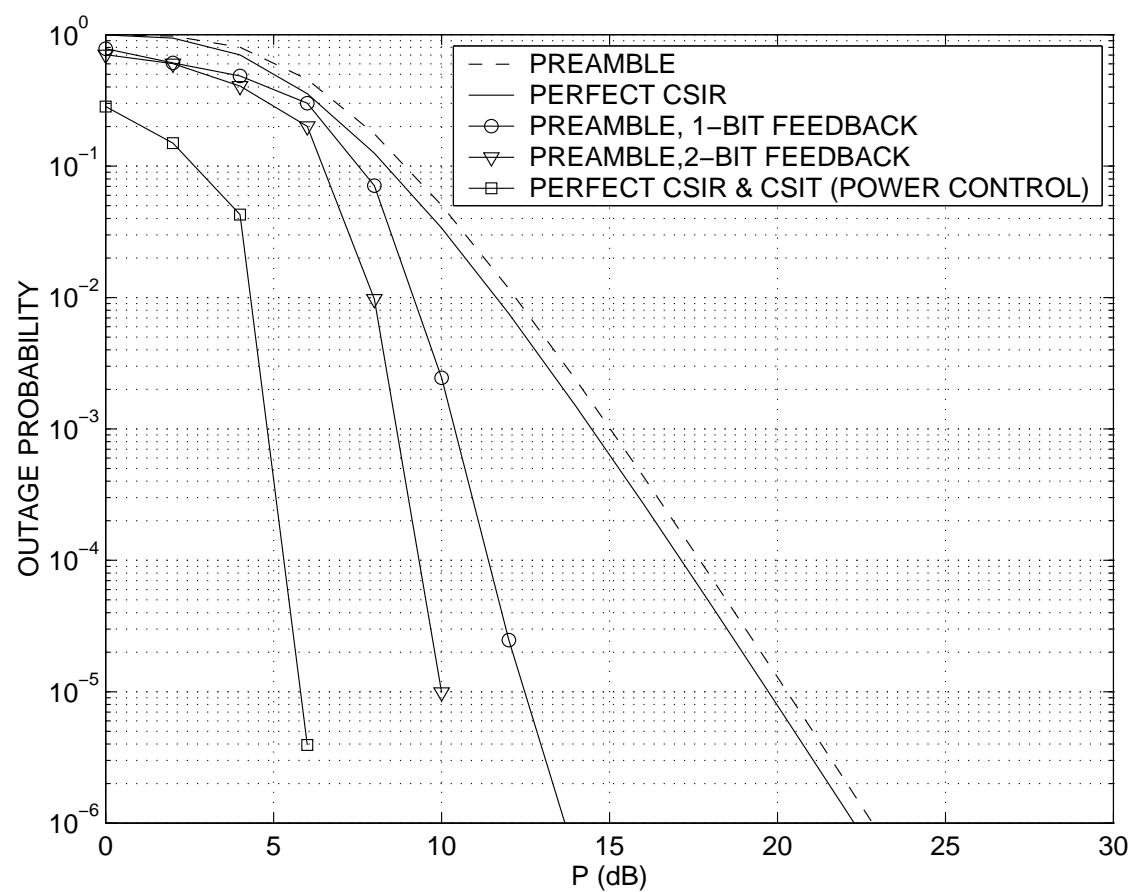

Fig. 8. Performance gains from 2-bit feedback - outage probability versus power constraint for $M=4$ transmit antennas, frame length $T=1000$, rate $R=2 \mathrm{bits} / \mathrm{s} / \mathrm{Hz}$.

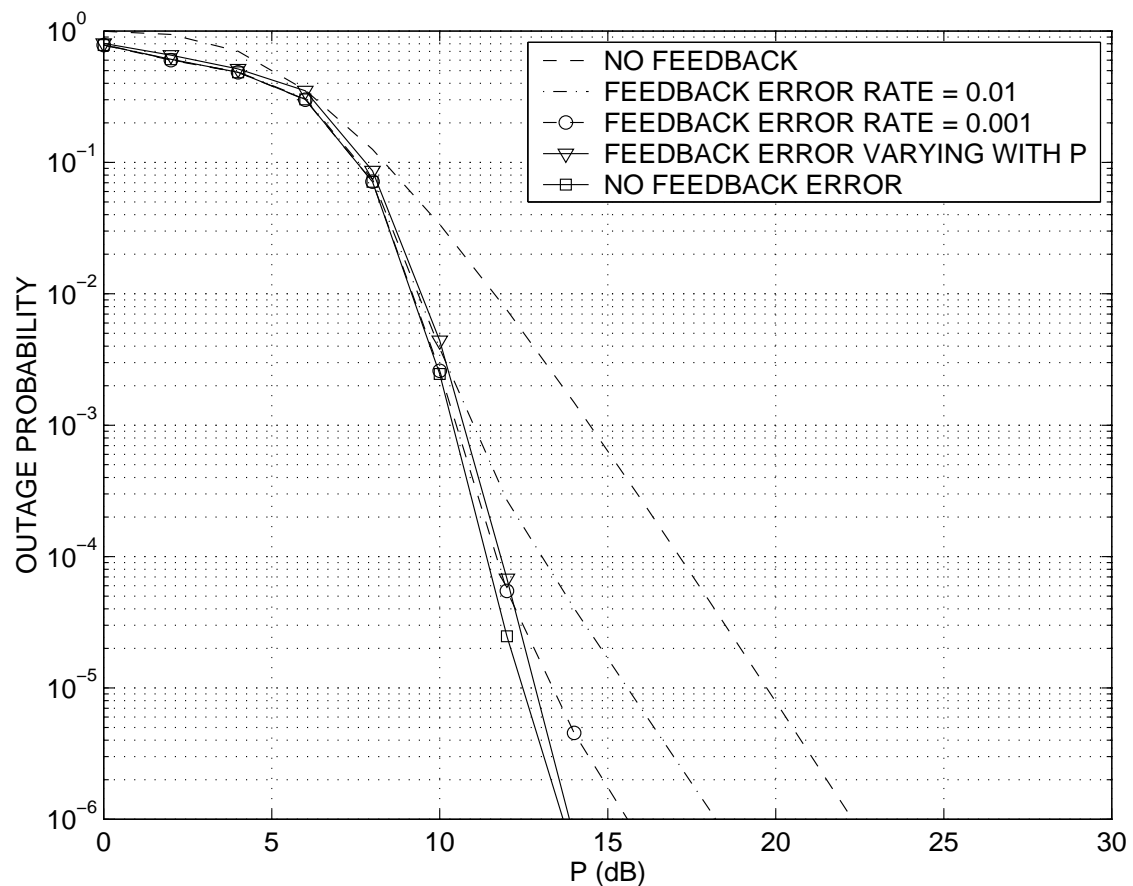

Fig. 9. Effect of feedback error on outage performance - outage probability versus power constraint for $M=4$ transmit antennas, frame length $T=1000$, rate $R=2 \mathrm{bits} / \mathrm{s} / \mathrm{Hz}$. 


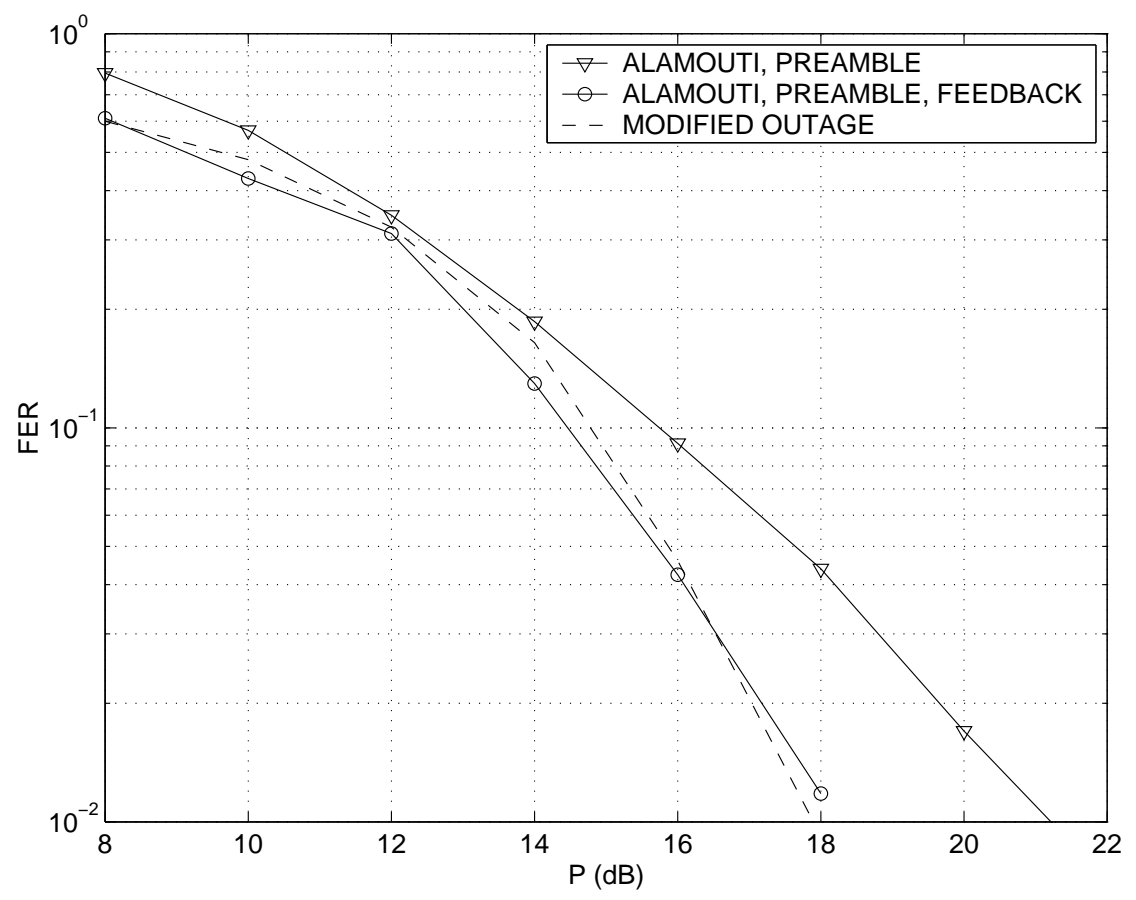

Fig. 10. Frame error rate of Alamouti scheme with feedback $-M=2$ transmit antennas, frame length $T=130$, rate $R=2 \mathrm{bits} / \mathrm{s} / \mathrm{Hz}$.

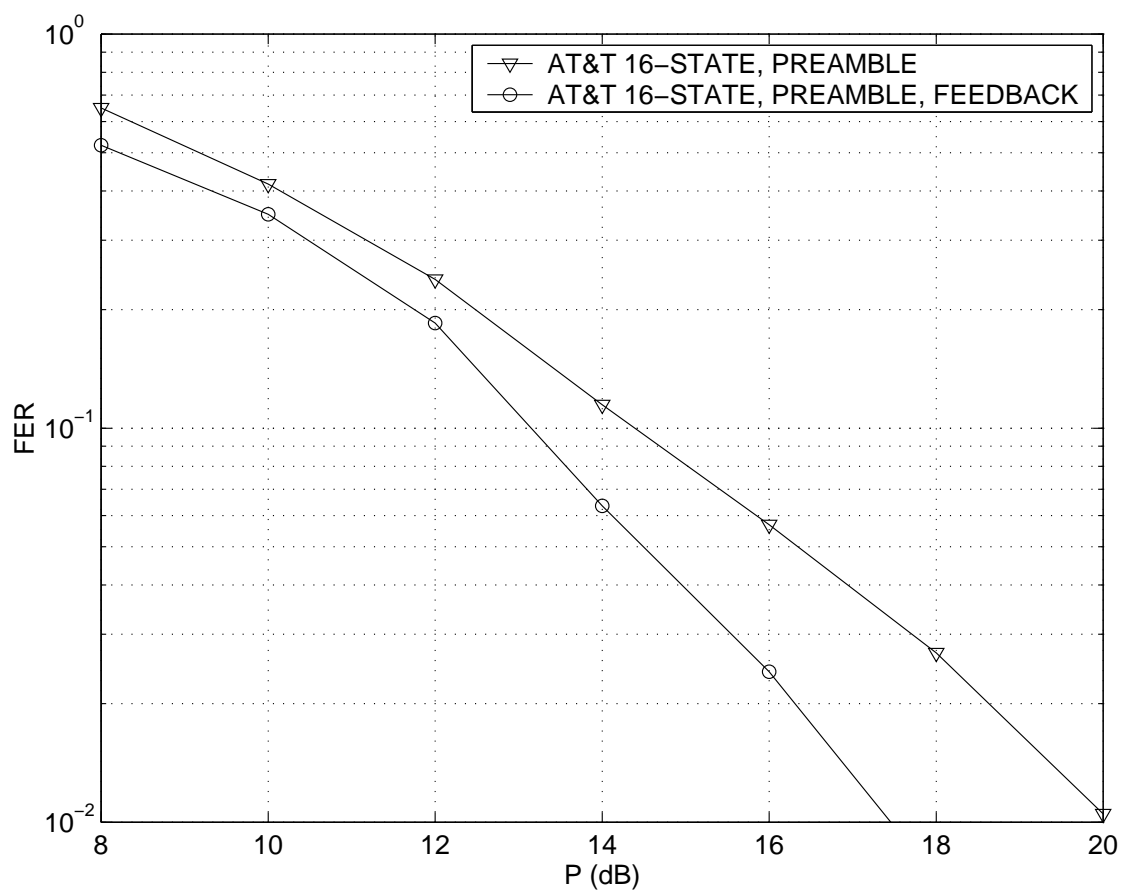

Fig. 11. Frame error rate of 16-state AT \& $\mathrm{T}$ space-time code with feedback $-M=2$ transmit antennas, frame length $T=130$, rate $R=2 \mathrm{bits} / \mathrm{s} / \mathrm{Hz}$. 